\title{
Compact planetary systems perturbed by an inclined companion: I. Vectorial representation of the secular model
}

\author{
Gwenaël Boué $e^{1,2}$ and Daniel C. Fabrycky ${ }^{1}$ \\ boue@uchicago.edu
}

\begin{abstract}
The non-resonant secular dynamics of compact planetary systems are modeled by a perturbing function which is usually expanded in eccentricity and absolute inclination with respect to the invariant plane. Here, the expressions are given in a vectorial form which naturally leads to an expansion in eccentricity and mutual inclination. The two approaches are equivalent in most cases, but the vectorial one is specially designed for those where a quasi-coplanar system tilts as a whole by a large amount. Moreover, the vectorial expressions of the Hamiltonian and of the equations of motion are slightly simpler than those given in terms of the usual elliptical elements. We also provide the secular perturbing function in vectorial form expanded in semimajor axis ratio allowing for arbitrary eccentricities and inclinations. The interaction between the equatorial bulge of a central star and its planets is also provided, as is the relativistic periapse precession of any planet induced by the central star. We illustrate the use of this representation for following the secular oscillations of the terrestrial planets of the solar system, and for Kozai cycles as may take place in exoplanetary systems.
\end{abstract}

Subject headings: methods: analytical — methods: numerical — celestial mechanics — planets and satellites: dynamical evolution and stability — planets and satellites: general — planet-star interactions

\section{Introduction}

Observations show nearly half of solar-type stars have a stellar companion (Duquennoy \& Mayor 1991; Raghavan et al. 2010) and close-in giant planets are found in such binary systems (Zucker \& Mazeh 2002, Udry \& Santos 2007). These observations have motivated extensive studies of the dynamics of a single planet evolving in a binary stellar system (Holman et al. 1997. Wu \& Murray 2003, Fabrycky \& Tremaine 2007. Wu et al. 2007; Correia et al. 2011; Naoz et al. 2011; Lithwick \& Naoz 2011; Katz et al. 2011, Veras \& Tout|2012 Kratter \& Perets |2012; $\mathrm{Naoz}$ et al. 2012, 2013a b). But above all, these systems

\footnotetext{
${ }^{1}$ Department of Astronomy and Astrophysics, University of Chicago, 5640 South Ellis Avenue, Chicago, IL 60637, USA

${ }^{2}$ Astronomie et Systèmes Dynamiques, IMCCE-CNRS UMR 8028, Observatoire de Paris, UPMC, 77 Av. DenfertRochereau, 75014 Paris, France.
}

present interesting dynamics due to the LidovKozai mechanism (Lidov 1962, Kozai 1962) which also provides a natural way to form hot Jupiters. The motion described by Lidov-Kozai dynamics takes place at large inclination and has the structure of a 1:1 resonance between the precession frequency of the longitude of pericenter $\varpi$ and of the longitude of the ascending node $\Omega$ of the planet. Inside the resonance, the critical angle, equal to the argument of the pericenter $\omega=\varpi-\Omega$, librates around either $90^{\circ}$ or $270^{\circ}$, and the eccentricity and inclination undergo large amplitude oscillations in antiphase. This behavior combined with tidal dissipation is able to shrink the orbit of a cold Jupiter down to orbital periods of about 3 days. During the evolution, the apsidal precession becomes dominated by general relativity, in which case the system exits the Lidov-Kozai resonance and the orbit evolves through tides as if the companion was nonexistent. The final obliquity of the star with respect to the planet orbit is often quite large 
(Fabrycky \& Tremaine 2007, Correia et al.|2011. Naoz et al. 2011, 2012, Li et al. 2014; Petrovich 2014). These theoretical predictions are supported by many observations of misaligned systems with a hot Jupiter (Winn et al. 2010; Triaud et al. 2010, Triaud 2011; Albrecht et al. 2012). The dynamical structure associated to the Lidov-Kozai mechanism subsists when the outer stellar companion is replaced by a planet (Terquem \& Papaloizou 2002 . Michtchenko et al. 2006; Libert \& Henrard 2007, 2008; Migaszewski \& Goździewski 2009; Mardling 2010; Naoz et al. 2011; Libert \& Delsate 2012). The outer planet only needs to be placed on a closer orbit than in the stellar case such that the secular timescale does not exceed the lifetime of the system. This framework has mainly been used to study the evolution and/or the formation of Jupiters on eccentric orbits. If both planets form in the same protoplanetary disk, the initial mutual inclination is expected to be small, but it can be generated by high order resonance crossings during planetary migration (Libert \& Tsiganis 2009, 2011), secular resonance overlaps (Wu \& Lithwick 2011), or planet-planet scattering (Nagasawa \& Ida 2011). Nevertheless, the latter evolutions are too violent for the Lidov-Kozai mechanism to play a significant role (Beaugé \& Nesvorný 2012). In multiplanet systems surrounded by an outer stellar companion, apsidal precession frequencies are dictated by the companion and by the planetplanet interactions. As a consequence, even at high inclination, if the planet system is sufficiently packed, planet-planet interactions dominate the apsidal motion, the evolution is stabilized with respect to the Lidov-Kozai mechanism, eccentricities remain small, and all planets move in concert (Innanen et al. 1997, Takeda et al. 2008, Saleh \& Rasio 2009). These systems are classified as dynamically rigid. Although the Lidov-Kozai evolution is quenched, the planetary mean plane still precesses if it is inclined relative to the orbit of the companion. The long term evolution of such systems can be followed with Laplace-Lagrange second order secular theory. This approach has already been applied in the context of planet formation in a binary system or of satellite formation around Uranus (Batygin et al. 2011; Batygin 2012, Morbidelli et al. 2012; Lai 2014). But higher order secular theories that assume absolute inclination remains small for all time can no longer follow the system. However, the secular dynamics should not simply be followed with Laplace-Lagrange secular model, which predicts quasiperiodic, bounded eccentricities and inclinations. For example, the solar system itself has chaotic secular dynamics (Laskar 1989, 1990), and only theories accurate to fourth or higher orders in eccentricities and inclinations are able to show the appearance of this chaos (Laskar 1984; Lithwick \& Wu 2011). The purpose of this paper is to provide a set of equations describing the secular evolution of nonresonant conservative gravitational systems with a massive central body. The formalism is very general and can be applied to many different types of systems. In a subsequent paper (Boué and Fabrycky 2014), we use it to study analytically the evolution of the spin-orbit angle in compact planetary systems perturbed by an inclined companion. Such analyses can a priori be performed numerically using an $n$-body integrator. However, the huge difference between orbital periods and secular timescales makes this approach hardly feasible in a reasonable amount of time. For instance, the 55 Cnc system (Fischer et al. 2008), a compact multiplanet system perturbed by a stellar companion (Mugrauer et al. 2006), contains five planets whose the innermost has a period of 0.73 days whereas the precession motion of the planetary system is about 70 Myr (Kaib et al. 2011, fig.1). The integration should thus last several hundreds of million years with a time step of a small fraction of a day. The long term evolution can also be followed with Gauss' method where the equations of motion, averaged over the mean longitudes of all planets, are given by double integrals (Touma et al. 2009). In practice, the first averaging is analytical while the second has to be computed numerically. This semi-analytic technique is faster than $n$-body codes and it can be applied to a large class of systems, even those with crossing orbits. Indeed, it does not assume any constraints on inclination, eccentricity, nor semimajor axis ratio. However, compact systems with large eccentricities or inclinations are likely to be unstable due to resonances overlap. One can thus assume that in most systems which are at least regular over a few secular timescales, each pair of planets is non-exclusively either hierarchical or quasi-coplanar with low eccentricities. These hypotheses are motivated by statistical studies 
of compact exoplanet systems detected by $\mathrm{Ke}$ pler or by radial velocity (e.g., Tremaine \& Dong 2012; Figueira et al. 2012, Fabrycky et al. 2012, Wu \& Lithwick 2013). Within this framework, planet-planet interactions are expanded either in semimajor axis ratio, or in mutual inclination and eccentricity. Hence, this technique is more restrictive than Gauss', but both the Hamiltonian and the equations of motion are analytical. This method is thus faster and more appropriate for analytical studies.

For this study, the expressions are given in a vectorial form which is independent of any reference frame. This approach, initiated by $\mathrm{Mi}-$ lankovitch (1939), has recently been proved very efficient in the study of cometary motion (Breiter \& Ratajczak 2005), of secular spin-orbit evolution and spin-spin interaction (Boué \& Laskar 2006. 2009), of the secular three-body problem (Farago \& Laskar 2010: Correia et al. 2011), and of the secular evolution of satellites (Tremaine et al. 2009, Tremaine \& Yavetz 2013). A detailed historical description of the construction of these variables and the associated equations of motion is given in Rosengren \& Scheeres (2014). The Hamiltonian and the equations of motion, derived in Section 2 . are expressed analytically by means of expansions in eccentricity and mutual inclination on the one hand, and in semimajor axis ratio, on the other. The independence of the vectorial equations from the reference frame is particularly useful for problems where the plane of a planet system tilts by a huge angle with respect to a given reference plane. This specific problem is treated in a subsequent paper (Boué \& Fabrycky, 2014). The model is tested in Section 3 against numerical integrations. The conclusions are given in the last section.

\section{Formalism}

Consider a system composed of an arbitrary number of massive bodies orbiting a central mass $m_{0}$. For simplicity, the central body is referred to as the central star and the others are called planets. Nevertheless, the formalism is more general and can be applied to other systems. For exemple, it can model stars orbiting a black hole, or satellite systems. We utilize Poincaré canonical astrocentric variables composed of astrocentric positions and barycentric velocities (e.g. Laskar \& Robu- tel 1995). Ellipses defined by these variables are not osculating, but for systems with more than three bodies, the formalism is simpler than that involving Jacobi coordinates. The canonical astrocentric variables (positions and conjugate momenta) of each body are noted $(\boldsymbol{r}, \tilde{\boldsymbol{r}})$, and the orbital elements $(a, \lambda, e, \varpi, I, \Omega)$ represent the semimajor axis, mean longitude, eccentricity, longitude of the pericenter, inclination, and longitude of the ascending node, respectively. All these elements are given with respect to a fixed reference frame. Whenever we consider two planets, quantities associated to the outermost are noted with a prime such as $m^{\prime}$. We also denote by $J$ the mutual inclination of any pairs of planets. In addition to the previous quantities, we also define $\beta=m_{0} m /\left(m_{0}+m\right), \mu=\mathcal{G}\left(m_{0}+m\right)$ where $\mathcal{G}$ is the universal gravitational constant, and $\Lambda=\beta \sqrt{\mu a}$. All quantities are recalled in Tab. 1 .

\subsection{Close-in interaction}

This section is devoted to the expansion of the perturbing function in inclination and eccentricity. The typical application of such this approximation is to model the interaction between two planets that are close to each other. In canonical astrocentric variables, the Hamiltonian describing the evolution of a compact pair of planets orbiting a central star

$$
H_{\text {close }}=K_{\text {close }}+H_{I, \text { close }}+H_{D, \text { close }}
$$

is the sum of a Keplerian part

$$
K_{\text {close }}=-\frac{\mu \beta}{2 a}-\frac{\mu^{\prime} \beta^{\prime}}{2 a^{\prime}},
$$

an indirect part

$$
H_{I, \text { close }}=\frac{\tilde{\boldsymbol{r}} \cdot \tilde{\boldsymbol{r}}^{\prime}}{m_{0}},
$$

and a direct part

$$
H_{D, \text { close }}=-\frac{\mathcal{G} m m^{\prime}}{a^{\prime}} \frac{a^{\prime}}{\Delta},
$$

where $\Delta=\left\|\boldsymbol{r}-\boldsymbol{r}^{\prime}\right\|$ is the distance between the two planets. Because we only focus on nonresonant secular evolutions, the indirect part $H_{I, \text { close }}$ of the Hamiltonian cancels and the Keplerian part $K_{\text {close }}$ remains constant. The secular evolution is thus solely controlled by the direct part $H_{D, \text { close }}$. 
Table 1: Notation.

\begin{tabular}{|c|c|c|c|}
\hline & variable & Ref. & description \\
\hline \multirow{27}{*}{ 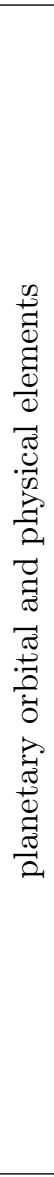 } & $\bar{r}, \boldsymbol{r}^{\prime}$ & & astrocentric position \\
\hline & $\tilde{\boldsymbol{r}}, \tilde{\boldsymbol{r}^{\prime}}$ & & barycentric velocity \\
\hline & $a, a^{\prime}$ & & semimajor axis \\
\hline & $\alpha$ & & semimajor axis ratio $a / a^{\prime}$ \\
\hline & $\lambda, \lambda^{\prime}$ & & mean longitude \\
\hline & $e, e^{\prime}$ & & eccentricity \\
\hline & $\omega, \omega^{\prime}$ & & argument of pericenter \\
\hline & $\varpi, \varpi^{\prime}$ & & longitude of pericenter \\
\hline & $I, I^{\prime}$ & & absolute inclination \\
\hline & $J$ & & mutual inclination between two planets \\
\hline & $\rho$ & & $\sin (J / 2)$ \\
\hline & $\sigma$ & & $\cos (J / 2)$ \\
\hline & $\Omega, \Omega^{\prime}$ & & longitude of the ascending node $\mathrm{ON}$ \\
\hline & $\tau$ & & $\mathrm{ON}+\mathrm{NG}$ \\
\hline & $\mathrm{O}$ & Fig. 1 ] & origin of longitude \\
\hline & $\mathrm{N}, \mathrm{N}^{\prime}$ & Fig. 1 & ascending node relative to the reference plane \\
\hline & $\mathrm{G}, \mathrm{G}^{\prime}$ & Fig. $\overline{1}$ & ascending node relative to another orbit plane \\
\hline & & & mutual distance $\left\|\boldsymbol{r}-\boldsymbol{r}^{\prime}\right\|$ \\
\hline & $m, m^{\prime}$ & & planet mass \\
\hline & $\beta, \beta^{\prime}$ & & reduced mass \\
\hline & $\mu, \mu^{\prime}$ & & $\mathcal{G}\left(m_{0}+m\right)$ \\
\hline & $\Lambda, \Lambda^{\prime}$ & & $\beta \sqrt{\mu a}$ \\
\hline & $e, e^{\prime}$ & & eccentricity vector \\
\hline & $\begin{array}{l}j, j^{\prime} \\
w, w^{\prime}\end{array}$ & & $\begin{array}{l}\text { dimensionless angular momentum } \sqrt{1-e^{2}} \boldsymbol{w} \\
\text { unit vector along the orbital angular momentum }\end{array}$ \\
\hline & $\xi, \xi^{\prime}$ & Eq. 15 & Souriau variable $j+e$ \\
\hline & $\boldsymbol{\eta}, \boldsymbol{\eta}^{\prime}$ & Eq. 15 & Souriau variable $\boldsymbol{j}-\boldsymbol{e}$ \\
\hline & $T_{j}, V_{j}, W_{j}$ & Eq. (18) & Abdullah variables quadratic in inclination and eccentricity \\
\hline \multirow{8}{*}{ 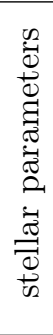 } & $m_{0}$ & & star mass \\
\hline & $R_{0}$ & & star radius \\
\hline & $C$ & & moment of inertia along the short axis \\
\hline & $k_{2}$ & & second fluid Love number \\
\hline & $J_{2}$ & Eq. 36 & quadrupole gravitational harmonic \\
\hline & $\omega_{0}$ & & rotation vector \\
\hline & $s$ & & spin axis \\
\hline & $L$ & & angular momentum $C \omega_{0} s$ \\
\hline \multirow{11}{*}{ 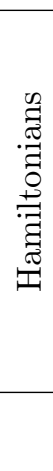 } & $H_{\text {close }}$ & Eq. 11 & Hamiltonian of packed system \\
\hline & $\bar{H}_{\text {close }}$ & Eq. 26 & secular Hamiltonian of packed system \\
\hline & $\bar{H}_{\text {hierar }}$ & Eq. 33 & secular Hamiltonian of hierarchical system \\
\hline & $\overline{\bar{H}}_{\text {spin }}$ & Eq. 34 & secular Hamiltonian of spin-orbit interaction \\
\hline & $\bar{H}_{\text {relat }}$ & Eq. (37) & secular Hamiltonian of general relativity \\
\hline & $\mathrm{b}_{s}^{(k)}(\alpha)$ & 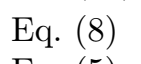 & Laplace coefficient \\
\hline & $f_{j}$ & Eq. 5 & coefficients of Le Verrier's expansion of the perturbing function \\
\hline & $g_{j}$ & Eq. 25 & coefficients of the perturbing function in Abdullah variables \\
\hline & $c_{j}$ & Tab. 3 & coefficients of the perturbing function in Milankovitch variables \\
\hline & $\mathcal{G}$ & & gravitational constant \\
\hline & $c$ & & speed of light \\
\hline
\end{tabular}




\subsubsection{Le Verrier's expansion}

The secular component of the expansion of $a^{\prime} / \Delta$ in eccentricity and absolute inclination is well known and is expressed analytically in, e.g., (Laskar \& Robutel 1995, Ellis \& Murray 2000). Although this approach is very convenient because the resulting expression is a polynomial in the canonical Poincaré variables (Laskar \& Robutel 1995), it has not been designed to study quasicoplanar systems with large absolute inclinations such as compact planetary systems perturbed by a distant and inclined stellar companion. One needs instead an expansion in mutual inclination as derived by Le Verrier (1855). The latter is simpler and more compact than expansions in absolute inclination because it is a special case where one of the absolute inclinations is set to zero. However, the equations of motion given in mutual inclination are more cumbersome, especially in systems with more than two planets. In the following, we recall the development of the secular component of $a^{\prime} / \Delta$ exact up to the fourth order in eccentricity $e, e^{\prime}$ and mutual inclination $J$. Then, we recall Le Verrier s equations of motion. The secular terms of $a^{\prime} / \Delta$ are

$$
\begin{aligned}
\left\langle\frac{a^{\prime}}{\Delta}\right\rangle_{\lambda, \lambda^{\prime}}= & f_{1} \\
& +f_{2}\left(e^{2}+e^{2}-4 \rho^{2}\right) \\
& +f_{3} e e^{\prime} \cos \left(\omega-\omega^{\prime}\right) \\
& +f_{4}\left(e^{2} e^{2}-4 \rho^{2}\left(e^{2}+e^{2}\right)\right) \\
& +f_{5} \rho^{2} e e^{\prime} \cos \left(\omega+\omega^{\prime}\right) \\
& +f_{6}\left(e^{4}+8 \rho^{2} e^{2} \cos 2 \omega^{\prime}\right) \\
& +f_{7}\left(e^{\prime 4}+8 \rho^{2} e^{2} \cos 2 \omega\right) \\
& +f_{8} \rho^{2} e e^{\prime} \cos \left(\omega-\omega^{\prime}\right) \\
& +f_{9} e^{3} e^{\prime} \cos \left(\omega-\omega^{\prime}\right) \\
& +f_{10} e e^{\prime 3} \cos \left(\omega-\omega^{\prime}\right) \\
& +f_{11} e^{2} e^{\prime 2} \cos 2\left(\omega-\omega^{\prime}\right) \\
& +f_{12} \rho^{4},
\end{aligned}
$$

with $\rho=\sin (J / 2)$. The secular Hamiltonian describing the evolution of a two planet system is then

$$
\bar{H}_{\text {close }}=-\frac{\mathcal{G} m m^{\prime}}{a^{\prime}}\left\langle\frac{a^{\prime}}{\Delta}\right\rangle_{\lambda, \lambda^{\prime}}
$$

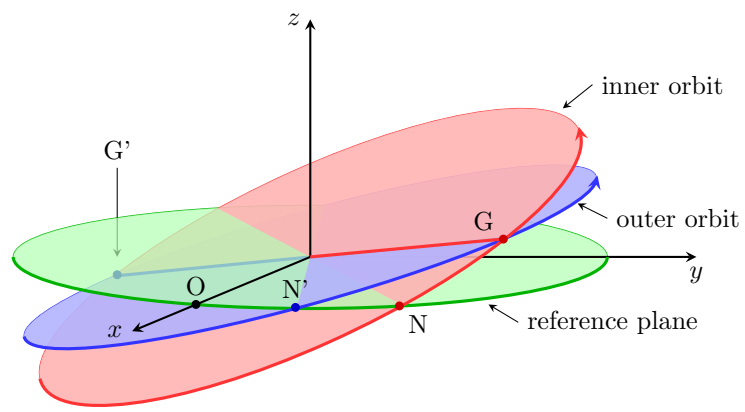

Fig. 1.- Orbit orientation. ON'N defines the reference plane. $\mathrm{O}$ is the origin of longitudes, $\mathrm{N}$ and $\mathrm{N}^{\prime}$ are the ascending nodes of the orbits of $m$ and $m^{\prime}$ relative to the reference plane, respectively. $\mathrm{G}$ and $\mathrm{G}^{\prime}$ are the ascending nodes of the orbit $m$ and $m^{\prime}$ relative to the orbit $m^{\prime}$ and $m$, respectively.

In (5), the orientation of the orbits are given relative to each other. More precisely, let $G$ be the ascending node of the inner orbit relative to the outer one, and similarly, G' the ascending node of the outer orbit with respect to the inner one. $\mathrm{G}$ and $\mathrm{G}^{\prime}$ are thus on the intersection between the two orbit planes, but in opposite directions (see Fig. 1). The angles $\omega$ and $\omega^{\prime}$ are the arguments of pericenter of the two planets relative to $G$ and $G$ ', respectively. With the notation of Fig. 1, the longitudes of the ascending nodes of $m$ and $m^{\prime}$ relative to the reference plane are $\Omega=\mathrm{ON}$ and $\Omega^{\prime}=\mathrm{ON}^{\prime}$, respectively. Following Le Verrier (1855), we denote $\tau=\mathrm{ON}+\mathrm{NG}$ and $\tau^{\prime}=\mathrm{ON}^{\prime}+\mathrm{N}^{\prime} \mathrm{G}^{\prime}$. The two arguments of pericenter $\omega$ and $\omega^{\prime}$ are then given by

$$
\begin{aligned}
& \omega=\varpi-\tau \\
& \omega^{\prime}=\varpi^{\prime}-\tau^{\prime} .
\end{aligned}
$$

Functions $\left(f_{k}\right)_{k=1, \ldots, 12}$ contain the dependency in the semimajor axis ratio $\alpha=a / a^{\prime}$. Le Verrier (1855) computed them in terms of Laplace coefficients $\mathrm{b}_{s}^{(k)}(\alpha)$, which are given by (e.g., Laskar \& Robutel 1995)

$$
\frac{1}{2} \mathrm{~b}_{s}^{(k)}(\alpha)=\frac{(s)_{k}}{k !} \alpha^{k} F\left(s, s+k, k+1 ; \alpha^{2}\right),
$$

and of their derivatives. In (8), $(s)_{0}=1$ and $(s)_{k}=s(s+1) \cdots(s+k-1)$ if $k>0$. The function $F(a, b, c ; x)$ is the Gauss hypergeometric function. Le Verrier's expressions of the functions $f_{k}$ 
are rather complicated. Here, we present instead those obtained using the algorithm described in Laskar \& Robutel (1995) and implemented in the algebraic manipulator TRIP (Gastineau \& Laskar 2012):

$$
\begin{aligned}
f_{1}= & \frac{1}{2} \mathrm{~b}_{1 / 2}^{(0)} \\
f_{2}= & \frac{1}{8} \alpha \mathrm{b}_{3 / 2}^{(1)} \\
f_{3}= & -\frac{3}{4} \alpha \mathrm{b}_{3 / 2}^{(0)}+\frac{1}{2}\left(1+\alpha^{2}\right) \mathrm{b}_{3 / 2}^{(1)}, \\
f_{4}= & \frac{9}{32} \alpha^{2} \mathrm{~b}_{5 / 2}^{(0)} \\
f_{5}= & \frac{9}{8} \alpha^{2} \mathrm{~b}_{5 / 2}^{(1)} \\
f_{6}= & -\frac{15}{128} \alpha^{2} \mathrm{~b}_{5 / 2}^{(0)}+\frac{3}{64} \alpha\left(1+3 \alpha^{2}\right) \mathrm{b}_{5 / 2}^{(1)} \\
f_{7}= & -\frac{15}{128} \alpha^{2} \mathrm{~b}_{5 / 2}^{(0)}+\frac{3}{64} \alpha\left(3+\alpha^{2}\right) \mathrm{b}_{5 / 2}^{(1)} \\
f_{8}= & -\frac{15}{4} \alpha\left(1+\alpha^{2}\right) \mathrm{b}_{5 / 2}^{(0)} \\
& +\frac{3}{4}\left(2+3 \alpha^{2}+2 \alpha^{4}\right) \mathrm{b}_{5 / 2}^{(1)}, \\
f_{9}= & -\frac{15}{16} \alpha \mathrm{b}_{5 / 2}^{(0)}+\frac{3}{32}\left(4+9 \alpha^{2}\right) \mathrm{b}_{5 / 2}^{(1)} \\
f_{10}= & -\frac{15}{16} \alpha^{3} \mathrm{~b}_{5 / 2}^{(0)}+\frac{3}{32} \alpha^{2}\left(9+4 \alpha^{2}\right) \mathrm{b}_{5 / 2}^{(1)}, \\
f_{11}= & \frac{45}{64} \alpha^{2} \mathrm{~b}_{5 / 2}^{(0)}-\frac{9}{32} \alpha\left(1+\alpha^{2}\right) \mathrm{b}_{5 / 2}^{(1)}, \\
f_{12}= & \frac{21}{8} \alpha^{2} \mathrm{~b}_{5 / 2}^{(0)}-\frac{3}{4} \alpha\left(1+\alpha^{2}\right) \mathrm{b}_{5 / 2}^{(1)} .
\end{aligned}
$$

In the averaged problem, the mean longitudes $\left(\lambda, \lambda^{\prime}\right)$ do not appear anymore, we thus discard their evolution. Furthermore, the semimajor axes are constant. The equations of motion derived by Le Verrier (1855) of the other orbital elements of any two planets $m$ and $m^{\prime}$ whatever are their po- sition relative to each other, are

$$
\begin{aligned}
\frac{d e}{d t}= & \frac{\sqrt{1-e^{2}}}{\Lambda e} \frac{\partial \bar{H}_{\text {close }}}{\partial \omega}, \\
\frac{d \varpi}{d t}= & -\frac{\sqrt{1-e^{2}}}{\Lambda e} \frac{\partial \bar{H}_{\text {close }}}{\partial e}+\tan \frac{I}{2} \sin I \frac{d \Omega}{d t}, \\
\frac{d I}{d t}= & \frac{\sin (\tau-\Omega)}{\Lambda \sqrt{1-e^{2}}} \frac{\partial \bar{H}_{\text {close }}}{\partial J} \\
& -\frac{\cos (\tau-\Omega)}{\Lambda \sqrt{1-e^{2}}}\left(f_{\tau}-\frac{\rho}{\sqrt{1-\rho^{2}}} \frac{\partial \bar{H}_{\text {close }}}{\partial \omega}\right), \\
\frac{d \Omega}{d t}= & -\frac{1}{\sin I}\left(\frac{\cos (\tau-\Omega)}{\Lambda \sqrt{1-e^{2}}} \frac{\partial \bar{H}_{\text {close }}}{\partial J}\right. \\
& \left.+\frac{\sin (\tau-\Omega)}{\Lambda \sqrt{1-e^{2}}}\left(f_{\tau}-\frac{\rho}{\sqrt{1-\rho^{2}}} \frac{\partial \bar{H}_{\text {close }}}{\partial \omega}\right)\right)
\end{aligned}
$$

with

$$
f_{\tau}=\frac{1}{\sin J}\left(\frac{\partial \bar{H}_{\text {close }}}{\partial \omega}+\frac{\partial \bar{H}_{\text {close }}}{\partial \omega^{\prime}}\right) .
$$

The relations between $(J, \tau)$ and the orbital elements relative to the reference plane are

$$
\begin{aligned}
& \cos J=\cos I \cos I^{\prime}+\sin I \sin I^{\prime} \cos \left(\Omega-\Omega^{\prime}\right), \\
& \sin (\tau-\Omega)=\frac{\sin I^{\prime}}{\sin J} \sin \left(\Omega-\Omega^{\prime}\right), \\
& \cos (\tau-\Omega)=\frac{\cos I^{\prime}-\cos I \cos J}{\sin I \sin J} .
\end{aligned}
$$

The above equations are sufficient to compute numerically the secular evolution of a $p$-planet system with low mutual inclinations and low eccentricities. However, they have singularities when any inclination or eccentricity is nil. This issue can be circumvented by choosing non singular variables such as $k=e \cos \varpi, h=e \sin \varpi$, $q=\sin (I / 2) \cos \Omega$, and $p=\sin (I / 2) \sin \Omega$, and by imposing $\dot{p}=\dot{q}=0$ whenever $\sin J=0$. In any case, the equations of motion (10) are complicated and do not facilitate the comprehension of the dynamical behavior of the system. For that reason, we shall consider a new set of variables.

\subsubsection{Milankovitch's variables}

The orientation in space and the shape of a Keplerian orbit (an ellipse) can be parametrized by 
two vectors: the dimensionless angular momentum $\boldsymbol{j}=\sqrt{1-e^{2}} \boldsymbol{w}$ and the Laplace-Runge-Lenz (or simply eccentricity) vector $\boldsymbol{e}=e \boldsymbol{u}$, where $\boldsymbol{w}$ is the unit vector normal to the orbit along the angular momentum, and $\boldsymbol{u}$ is the unit vector pointing toward the pericenter. These vectors are used in place of the usual elliptical elements $(e, \varpi, I, \Omega)$. Since each vector has three coordinates, the number of variables increases from four to six. This implies that the new variables are not independent, and indeed, they are related by two equations:

$$
\boldsymbol{e} \cdot \boldsymbol{j}=0, \quad\|\boldsymbol{e}\|^{2}+\|\boldsymbol{j}\|^{2}=1 .
$$

The equations of motion expressed in terms of these two vectors were derived by Milankovitch (1939). In the secular problem, they read as (e.g., Breiter \& Ratajczak 2005; Tremaine et al. 2009, Rosengren \& Scheeres 2014)

$$
\begin{aligned}
& \frac{d \boldsymbol{j}}{d t}=-\frac{1}{\Lambda}\left(\boldsymbol{j} \times \nabla_{\boldsymbol{j}} \bar{H}+\boldsymbol{e} \times \nabla_{\boldsymbol{e}} \bar{H}\right), \\
& \frac{d \boldsymbol{e}}{d t}=-\frac{1}{\Lambda}\left(\boldsymbol{e} \times \nabla_{\boldsymbol{j}} \bar{H}+\boldsymbol{j} \times \nabla_{\boldsymbol{e}} \bar{H}\right),
\end{aligned}
$$

where $\bar{H}$ (here $\bar{H}=\bar{H}_{\text {close }}$ ) is the secular Hamiltonian of the system written in terms of the vectors $\left(\boldsymbol{e}, \boldsymbol{j}, \boldsymbol{e}^{\prime}, \boldsymbol{j}^{\prime}\right)$. The variables $(\boldsymbol{e}, \boldsymbol{j})$ are not singular. Furthermore, they lead to more compact and symmetrical equations to describe the evolution of the system. The expression of the Hamiltonian as a function of these vectors is presented in the following section.

\subsubsection{Souriau's variables}

This section reproduces the computation of the perturbing function (6) made by Abdullah (2001) in terms of the vectors $e$ and $j^{1}$. This method involves new variables named after Souriau (1969). These variables noted $\boldsymbol{\xi}$ and $\boldsymbol{\eta}$, are defined by

$$
\begin{gathered}
\boldsymbol{\xi}=\boldsymbol{j}+\boldsymbol{e} \\
\boldsymbol{\eta}=\boldsymbol{j}-\boldsymbol{e}
\end{gathered}
$$

It can easily be shown that

$$
\|\boldsymbol{\xi}\|=1 \quad\|\boldsymbol{\eta}\|=1
$$

\footnotetext{
${ }^{1}$ Our expressions differ slightly from those obtained by Abdullah (2001) because we use $\rho=\sin (J / 2)$ like in the work of Le Verrier (1855), whereas in Abdullah s notation, $\rho=\sin J$. Furthermore, the scaling factor in Laplace coefficients is arbitrary, and Abdullah (2001) do not include the factor $1 / 2$ that we have in $(8)$.
}

Relations 16 let Souriau (1969) conclude that the set of ellipses with one fixed focus and semimajor axis is equivalent to the product of two spheres: $S^{2} \times S^{2}$. The dimension of the problem is thus clearly four. From (14) and (15), the derivation of the equations of motion of $\boldsymbol{\xi}$ and $\boldsymbol{\eta}$ is straightforward. The result is

$$
\begin{aligned}
& \frac{d \boldsymbol{\xi}}{d t}=-\frac{2}{\Lambda} \boldsymbol{\xi} \times \nabla_{\boldsymbol{\xi}} H, \\
& \frac{d \boldsymbol{\eta}}{d t}=-\frac{2}{\Lambda} \boldsymbol{\eta} \times \nabla_{\boldsymbol{\eta}} H .
\end{aligned}
$$

In these variables, the equations of motion (17) are very simple and symmetric. To get the expression of the secular Hamiltonian as a function of Souriau's variables, one needs to expand $\left\langle a^{\prime} / \Delta\right\rangle$ in terms of these variables, but since their norms are equal to one, by construction, they are not small quantities. To solve that issue, Abdullah (2001) considered a new family of variables based on Souriau's one, and defined by

$$
\begin{aligned}
& T_{1}=\frac{1}{2}(1-\boldsymbol{\xi} \cdot \boldsymbol{\eta}), \\
& T_{2}=\frac{1}{2}\left(1-\boldsymbol{\xi}^{\prime} \cdot \boldsymbol{\eta}^{\prime}\right), \\
& V_{1}=\frac{1}{2}\left(1-\boldsymbol{\xi} \cdot \boldsymbol{\xi}^{\prime}\right), \\
& V_{2}=\frac{1}{2}\left(1-\boldsymbol{\eta} \cdot \boldsymbol{\eta}^{\prime}\right), \\
& W_{1}=\frac{1}{2}\left(1-\boldsymbol{\xi} \cdot \boldsymbol{\eta}^{\prime}\right), \\
& W_{2}=\frac{1}{2}\left(1-\boldsymbol{\xi}^{\prime} \cdot \boldsymbol{\eta}\right) .
\end{aligned}
$$

These variables can be interpreted as the square of the semi-distances between the points represented on the unit sphere by the vectors $\boldsymbol{\xi}, \boldsymbol{\xi}^{\prime}, \boldsymbol{\eta}$, and $\boldsymbol{\eta}^{\prime}$. Indeed, for instance,

$$
T_{1}=\left(\frac{1}{2}\|\boldsymbol{\xi}-\boldsymbol{\eta}\|\right)^{2}
$$

The variables (18) are quadratic in eccentricities and in mutual inclinations. The method is then to express all the quantities appearing in the expression (6), i.e., $\rho^{2}, e^{2}, e^{\prime 2}, p(k, \ell)$ and $q(k, \ell)$ as a function of the variables $\sqrt{18}$, where $p(k, \ell)$ and $q(k, \ell)$ are defined for all $(k, \ell) \in \mathbb{N}^{2}$ by

$$
\begin{aligned}
& p(k, \ell)=\rho^{k+\ell} e^{k} e^{\ell \ell} \cos \left(k \omega+\ell \omega^{\prime}\right), \\
& q(k, \ell)=\rho^{|k-\ell|} e^{k} e^{\ell \ell} \cos \left(k \omega-\ell \omega^{\prime}\right),
\end{aligned}
$$


if $k+\ell$ is even, and

$$
\begin{aligned}
& p(k, \ell)=\rho^{k+\ell} e^{k} e^{\ell \ell} \sin \left(k \omega+\ell \omega^{\prime}\right), \\
& q(k, \ell)=\rho^{|k-\ell|} e^{k} e^{\prime \ell} \sin \left(k \omega-\ell \omega^{\prime}\right),
\end{aligned}
$$

if $k+\ell$ is odd. To simplify the substitution, Abdullah (2001) provides recurrence relations to compute the $p(k, \ell)$ and $q(k, \ell)$ which are equivalent to those of Table 2. To initiate the recurrence, we have

$$
\begin{aligned}
e^{2} & =T_{1}, \\
e^{2} & =T_{2} \\
\rho^{2} & =\frac{1}{2}-\frac{2-V_{1}-V_{2}-W_{1}-W_{2}}{4 \sqrt{\left(1-T_{1}\right)\left(1-T_{2}\right)}}, \\
p(1,0) & =\frac{V_{2}-V_{1}+W_{2}-W_{1}}{4 \sqrt{\left(1-T_{2}\right)\left(1-\rho^{2}\right)}} \\
p(0,1) & =\frac{V_{2}-V_{1}+W_{1}-W_{2}}{4 \sqrt{\left(1-T_{1}\right)\left(1-\rho^{2}\right)}} \\
q(1,1) & =\frac{V_{1}+V_{2}-W_{1}-W_{2}}{2} \\
& +2 p(1,0) p(0,1) \\
p(1,1) & =\rho^{2} \frac{V_{1}+V_{2}-W_{1}-W_{2}}{2} \\
& -2\left(1-\rho^{2}\right) p(1,0) p(0,1),
\end{aligned}
$$

and

$$
\begin{aligned}
& q(k, 0)=p(k, 0), \\
& q(0, \ell)=-p(0, \ell)
\end{aligned}
$$

for all $(k, \ell)$ in $\mathbb{N}^{2}$. After substituting the terms $p(k, \ell)$ and $q(k, \ell)$ in (5) by $T_{1}, T_{2}, V_{1}, V_{2}, W_{1}$, and $W_{2}$, and truncating at the second order in these new variables, Abdullah (2001) obtained

$$
\begin{aligned}
\left\langle\frac{a^{\prime}}{\Delta}\right\rangle_{\lambda, \lambda^{\prime}} & =f_{1} \\
& +f_{2}\left(2 T_{1}+2 T_{2}-V_{1}-V_{2}-W_{1}-W_{2}\right) \\
& +\frac{1}{2} f_{3}\left(V_{1}+V_{2}-W_{1}-W_{2}\right) \\
& +g_{4}\left(\alpha^{2} T_{1}^{2}+T_{2}^{2}\right) \\
& +g_{5}\left(V_{1}^{2}+V_{2}^{2}\right) \\
& +g_{6}\left(W_{1}^{2}+W_{2}^{2}\right) \\
& +g_{7}\left(T_{1} T_{2}+V_{1} V_{2}+W_{1} W_{2}\right) \\
& +g_{8}\left(\alpha T_{1}-T_{2}\right)\left(V_{1}+V_{2}\right) \\
& +g_{9}\left(\alpha T_{1}+T_{2}\right)\left(W_{1}+W_{2}\right) \\
& +g_{10}\left(V_{1} W_{1}+V_{2} W_{2}\right) \\
& +g_{11}\left(V_{1} W_{2}+V_{2} W_{1}\right)
\end{aligned}
$$

where $f_{1}, f_{2}$, and $f_{3}$ are given in $(9)$ and

$$
\begin{aligned}
& g_{4}=\frac{9}{32} \alpha \mathrm{b}_{5 / 2}^{(1)} \\
& g_{5}=\frac{3}{16} \alpha\left(-5+4 \alpha-5 \alpha^{2}\right) \mathrm{b}_{5 / 2}^{(0)}+\frac{3}{32}\left(2-\alpha+2 \alpha^{2}\right)^{2} \mathrm{~b}_{5 / 2}^{(1)} \\
& g_{6}=\frac{3}{16} \alpha\left(5+4 \alpha+5 \alpha^{2}\right) \mathrm{b}_{5 / 2}^{(0)}-\frac{3}{32}\left(2+\alpha+2 \alpha^{2}\right)^{2} \mathrm{~b}_{5 / 2}^{(1)} \\
& g_{7}=\frac{9}{16} \alpha^{2} \mathrm{~b}_{5 / 2}^{(0)} \\
& g_{8}=-\frac{15}{32} \alpha(1-\alpha) \mathrm{b}_{5 / 2}^{(0)}+\frac{3}{16}\left(1-\alpha^{3}\right) \mathrm{b}_{5 / 2}^{(1)} \\
& g_{9}=-\frac{15}{32} \alpha(1+\alpha) \mathrm{b}_{5 / 2}^{(0)}+\frac{3}{16}\left(1+\alpha^{3}\right) \mathrm{b}_{5 / 2}^{(1)} \\
& g_{10}=-\frac{3}{8} \alpha^{2}\left(\mathrm{~b}_{5 / 2}^{(0)}-\alpha \mathrm{b}_{5 / 2}^{(1)}\right) \\
& g_{11}=-\frac{3}{8} \alpha\left(\alpha \mathrm{b}_{5 / 2}^{(0)}-\mathrm{b}_{5 / 2}^{(1)}\right)
\end{aligned}
$$

The expansion (24) can be seen as a function of Souriau's variables $\left(\boldsymbol{\xi}, \boldsymbol{\eta}, \boldsymbol{\xi}^{\prime}, \boldsymbol{\eta}^{\prime}\right)$ using (18), or as a function of Milankovitch's variables $\left(\boldsymbol{e}, \boldsymbol{j}, \boldsymbol{e}^{\prime}, \boldsymbol{j}^{\prime}\right)$ using the definition of Souriau's variables 15. 
Table 2: Recurrence relations for the computation of $p(k, \ell)$ and $q(k, \ell)$, Eqs. (20)-21).

\begin{tabular}{|c|c|}
\hline$p(k, \ell)=2 p(k-1, \ell-1) p(1,1)-\rho^{4} e^{2} e^{\prime 2} p(k-2, \ell-2)$ & $k \geq 2$ and $\ell \geq 2$ \\
\hline$p(k, 1)=2 p(k-1,0) p(1,1)-\rho^{4} e^{2} q(k-2,1)$ & $k>2$ \\
\hline$p(1, \ell)=2 p(0, \ell-1) p(1,1)+(-1)^{\ell} \rho^{4} e^{\prime 2} q(1, \ell-2)$ & $\ell>2$ \\
\hline$p(k, 0)=2(-1)^{k+1} p(k-1,0) p(1,0)+\rho^{2} e^{2} p(k-2,0)$ & $k \geq 2$ \\
\hline$p(0, \ell)=2(-1)^{\ell+1} p(0, \ell-1) p(0,1)+\rho^{2} e^{\prime 2} p(0, \ell-2)$ & $\ell \geq 2$ \\
\hline \multicolumn{2}{|l|}{$p(2,1)=2 p(1,0) p(1,1)-\rho^{2} e^{2} q(0,1)$} \\
\hline \multicolumn{2}{|l|}{$p(1,2)=2 p(0,1) p(1,1)+\rho^{2} e^{\prime 2} q(1,0)$} \\
\hline$q(k, \ell)=2 q(k-1, \ell-1) q(1,1)-e^{2} e^{\prime 2} q(k-2, \ell-2)$ & $k \geq 2$ and $\ell \geq 2$ \\
\hline$q(k, 1)=2 q(k-1,0) q(1,1)-e^{2} p(k-2,1)$ & $k \geq 2$ \\
\hline$q(1, \ell)=2 q(0, \ell-1) q(1,1)+(-1)^{\ell} e^{\prime 2} p(1, \ell-2)$ & $\ell \geq 2$ \\
\hline$q(k, 0)=p(k, 0), \quad q(0, \ell)=(-1)^{\ell} p(0, \ell)$ & $\forall k, l$ \\
\hline
\end{tabular}

One gets

$$
\begin{aligned}
\left\langle\frac{a^{\prime}}{\Delta}\right\rangle_{\lambda, \lambda^{\prime}} & =c_{1} \\
& +c_{2}\left(e^{2}+e^{2}+\boldsymbol{j} \cdot \boldsymbol{j}^{\prime}-1\right) \\
& +c_{3}\left(\boldsymbol{e} \cdot \boldsymbol{e}^{\prime}\right) \\
& +c_{4} e^{2} e^{\prime 2} \\
& +c_{5}\left(\alpha^{2} e^{4}+e^{44}\right) \\
& +c_{6}\left(\boldsymbol{e} \cdot \boldsymbol{e}^{\prime}\right)^{2} \\
& +c_{7}\left(1-\boldsymbol{j} \cdot \boldsymbol{j}^{\prime}\right)^{2} \\
& +c_{8}\left(\boldsymbol{e} \cdot \boldsymbol{j}^{\prime}\right)^{2} \\
& +c_{9}\left(\boldsymbol{j} \cdot \boldsymbol{e}^{\prime}\right)^{2} \\
& +c_{10}\left(\alpha\left(1-\boldsymbol{j} \cdot \boldsymbol{j}^{\prime}\right) e^{2}+\left(\boldsymbol{e} \cdot \boldsymbol{e}^{\prime}\right) e^{2}\right) \\
& +c_{11}\left(\left(1-\boldsymbol{j} \cdot \boldsymbol{j}^{\prime}\right) e^{2}+\alpha\left(\boldsymbol{e} \cdot \boldsymbol{e}^{\prime}\right) e^{2}\right) \\
& +c_{12}\left(\left(1-\boldsymbol{j} \cdot \boldsymbol{j}^{\prime}\right)\left(\boldsymbol{e} \cdot \boldsymbol{e}^{\prime}\right)\right. \\
& \left.-\left(\boldsymbol{e} \cdot \boldsymbol{j}^{\prime}\right)\left(\boldsymbol{j} \cdot \boldsymbol{e}^{\prime}\right)\right)
\end{aligned}
$$

The first three lines with coefficients $c_{1}, c_{2}$, and $c_{3}$ correspond to the second order expansion in eccentricity and mutual inclination. All the other terms are associated to the order 4 . Because the dot product of two vectors is invariant by any rotation applied on both vectors, the expression 26 . is clearly invariant by rotation. Thus, the refer- ence frame does not need to be aligned with the mean plane of the planet system. The coefficients $\left(c_{k}\right)_{k=1, \cdots, 12}$ are

$$
\begin{aligned}
& c_{1}=f_{1}, \\
& c_{2}=2 f_{2}, \\
& c_{3}=-f_{3}, \\
& c_{4}=g_{7}, \\
& c_{5}=g_{4}, \\
& c_{6}=\frac{1}{2}\left(g_{5}+g_{6}+g_{7}-g_{10}-g_{11}\right), \\
& c_{7}=\frac{1}{2}\left(g_{5}+g_{6}+g_{7}+g_{10}+g_{11}\right), \\
& c_{8}=\frac{1}{2}\left(g_{5}+g_{6}-g_{7}+g_{10}-g_{11}\right), \\
& c_{9}=\frac{1}{2}\left(g_{5}+g_{6}-g_{7}-g_{10}+g_{11}\right), \\
& c_{10}=g_{9}+g_{8}, \\
& c_{11}=g_{9}-g_{8}, \\
& c_{12}=g_{6}-g_{5} .
\end{aligned}
$$

Their explicit expressions in terms of Laplace coefficients are given in Tab3 The equations of motion, derived from (6), (14), and (26), are written in Appendix A.1. We stress here that the vectorial expansion (26) is a generalization of the more standard development in eccentricity and absolute 
Table 3: Coefficients of the secular expansion of the perturbing function in eccentricity and mutual inclination.

$$
\begin{aligned}
c_{1} & =\frac{1}{2} \mathrm{~b}_{1 / 2}^{(0)} \\
c_{2} & =\frac{1}{4} \alpha \mathrm{b}_{3 / 2}^{(1)} \\
c_{3} & =\frac{3}{4} \alpha \mathrm{b}_{3 / 2}^{(0)}-\frac{1}{2}\left(1+\alpha^{2}\right) \mathrm{b}_{3 / 2}^{(1)} \\
c_{4} & =\frac{9}{16} \alpha^{2} \mathrm{~b}_{5 / 2}^{(0)} \\
c_{5} & =\frac{9}{32} \alpha \mathrm{b}_{5 / 2}^{(1)} \\
c_{6} & =\frac{45}{32} \alpha^{2} \mathrm{~b}_{5 / 2}^{(0)}-\frac{9}{16} \alpha\left(1+\alpha^{2}\right) \mathrm{b}_{5 / 2}^{(1)} \\
c_{7} & =\frac{21}{32} \alpha^{2} \mathrm{~b}_{5 / 2}^{(0)}-\frac{3}{16} \alpha\left(1+\alpha^{2}\right) \mathrm{b}_{5 / 2}^{(1)} \\
c_{8} & =\frac{15}{32} \alpha^{2} \mathrm{~b}_{5 / 2}^{(0)}-\frac{3}{16} \alpha\left(3+\alpha^{2}\right) \mathrm{b}_{5 / 2}^{(1)} \\
c_{12} & =\frac{15}{32} \alpha^{2} \mathrm{~b}_{5 / 2}^{(0)}-\frac{3}{16} \alpha\left(1+3 \alpha^{2}\right) \mathrm{b}_{5 / 2}^{(1)} \\
c_{11} & \left.=-\frac{15}{16} \alpha^{2} \mathrm{~b}_{5 / 2}^{(0)}+\frac{3}{8} \alpha^{3} \mathrm{~b}_{5 / 2}^{(1)}+\alpha^{2}\right) \mathrm{b}_{5 / 2}^{(0)}-\frac{3}{16}\left(4+9 \alpha^{2}+4 \alpha^{4}\right) \mathrm{b}_{5 / 2}^{(1)} \\
c_{10} & =-\frac{3}{16} \alpha \mathrm{b}_{5 / 2}^{(0)}+\frac{3}{8} \mathrm{~b}_{5 / 2}^{(1)} \\
c_{5} &
\end{aligned}
$$

inclination (e.g., Laskar \& Robutel 1995, Ellis \& Murray 2000). Indeed, at low inclination with respect to the reference frame, both formalisms are equivalent. If the planet system is tilted by a large angle, the linear equations of the LaplaceLagrange approximation are still valid. But at higher orders, where inclinations get coupled with eccentricities, tilted systems can only be described with expansions in mutual inclination such as in the vectorial approach.

\subsection{Hierarchical interaction}

Consider the case where the two planets $m, m^{\prime}$ are very distant from each other $\left(\alpha \equiv a / a^{\prime} \lesssim 0.1\right)$. The Hamiltonian $\bar{H}_{\text {hierar }}$ governing the secular evolution is similar to $\bar{H}_{\text {close }}$ (6),

$$
\bar{H}_{\text {hierar }}=-\frac{\mathcal{G} m m^{\prime}}{a^{\prime}}\left\langle\frac{a^{\prime}}{\Delta}\right\rangle_{\lambda, \lambda^{\prime}} .
$$

The only difference is that $\left\langle a^{\prime} / \Delta\right\rangle$ is expanded in semimajor axis ratio $\alpha$ rather than in eccentricity and inclination. The most important contributions to this development have been made in the XIXth century (Hansen 1853; Hill 1875; Tisserand 1889). The explicit expression of the secular perturbing function expanded at the octupolar order 
is (e.g., Laskar \& Boué 2010

$$
\begin{aligned}
\left\langle\frac{a^{\prime}}{\Delta}\right\rangle_{\lambda, \lambda^{\prime}} & =1+\left(\left(\frac{1}{4}-\frac{3}{2} \rho^{2}+\frac{3}{2} \rho^{4}\right)\left(1+\frac{3}{2} e^{2}\right)\right. \\
& \left.+\frac{15}{4} \rho^{2} \sigma^{2} e^{2} \cos (2 \omega)\right) \frac{\alpha^{2}}{\left(1-e^{2}\right)^{3 / 2}} \\
& +\frac{15}{16}\left(\left(1+\frac{3}{4} e^{2}\right)\right. \\
& \times\left[\sigma^{2}\left(1-10 \rho^{2}+15 \rho^{4}\right) \cos \left(\omega-\omega^{\prime}\right)\right. \\
& \left.+\rho^{2}\left(6-20 \rho^{2}+15 \rho^{4}\right) \cos \left(\omega+\omega^{\prime}\right)\right] \\
& +\frac{35}{4} e^{2} \rho^{2} \sigma^{2}\left[\rho^{2} \cos \left(3 \omega+\omega^{\prime}\right)\right. \\
& \left.\left.+\sigma^{2} \cos \left(3 \omega-\omega^{\prime}\right)\right]\right) \frac{e e^{\prime} \alpha^{3}}{\left(1-e^{\prime 2}\right)^{5 / 2}}
\end{aligned}
$$

with $\sigma^{2}=1-\rho^{2}$. As in section 2.1, one can use the equations of motion (10) derived by Le Verrier (1855) to get the evolution of the system described by (29), but once again, the expressions are much simpler in a vectorial form. To get the vectorial expression of $\left\langle a^{\prime} / \Delta\right\rangle$ expanded in semimajor axis ratio, we follow an algorithm very similar to the one of the section 2.1. We define $P(k, \ell)$ and $Q(k, \ell)$, $(k, \ell) \in \mathbb{N}^{2}$, as

$$
\begin{aligned}
& P(k, \ell)=\sigma^{|k-\ell|} p(k, \ell), \\
& Q(k, \ell)=\sigma^{k+\ell} q(k, \ell),
\end{aligned}
$$

where $p(k, \ell)$ and $q(k, \ell)$ are given in Eqs (20)-(21). The secular part of the expansion in semimajor axis ratio of the perturbing function is a polynomial in $\alpha, \rho^{2}, e^{2}, e^{\prime 2},\left(1-e^{\prime 2}\right)^{-1 / 2}, P(k, \ell)$, and $Q(k, \ell)$ (Tisserand 1889). The recurrence relations satisfied by $P(k, \ell)$ and $Q(k, \ell)$ are displayed in Table 4 . The initialization of the recurrence can be

\footnotetext{
${ }^{2}$ In this paper, $\omega^{\prime}$ is defined with respect to the ascending node G' of the orbit $m^{\prime}$ relative to the orbit $m$, while in Laskar \& Boué (2010), $\omega^{\prime}$ is defined with respect to the ascending node $\mathrm{G}$ of the orbit $m$ relative to the orbit $m^{\prime}$. Thus, the two arguments of periastron differ by $\pi$.
}

done either in terms of Souriau's variables

$$
\begin{aligned}
e^{2}= & T_{1}, \\
e^{\prime 2}= & T_{2}, \\
\rho^{2}= & \frac{1}{2}-\frac{2-V_{1}-V_{2}-W_{1}-W_{2}}{4 \sqrt{\left(1-T_{1}\right)\left(1-T_{2}\right)}}, \\
P(1,0)= & \frac{V_{2}-V_{1}+W_{2}-W_{1}}{4 \sqrt{1-T_{2}}}, \\
P(0,1)= & \frac{V_{2}-V_{1}+W_{1}-W_{2}}{4 \sqrt{1-T_{1}}}, \\
Q(1,1)= & \sigma^{2} \frac{V_{1}+V_{2}-W_{1}-W_{2}}{2} \\
& +2 P(1,0) P(0,1), \\
P(1,1)= & \rho^{2} \frac{V_{1}+V_{2}-W_{1}-W_{2}}{2} \\
& -2 P(1,0) P(0,1),
\end{aligned}
$$

or directly in terms of Milankovitch's vectors

$$
\begin{aligned}
& 2 \rho^{2}=1-\boldsymbol{w} \cdot \boldsymbol{w}^{\prime}, \\
& 2 \sigma^{2} \quad=1+\boldsymbol{w} \cdot \boldsymbol{w}^{\prime}, \\
& 2 P(1,0)=\left(\boldsymbol{w}^{\prime} \cdot \boldsymbol{e}\right), \\
& 2 P(0,1)=\left(\boldsymbol{w} \cdot \boldsymbol{e}^{\prime}\right), \\
& 2 Q(1,1)=-2 \sigma^{2}\left(\boldsymbol{e} \cdot \boldsymbol{e}^{\prime}\right)+\left(\boldsymbol{e} \cdot \boldsymbol{w}^{\prime}\right)\left(\boldsymbol{w} \cdot \boldsymbol{e}^{\prime}\right), \\
& 2 P(1,1)=-2 \rho^{2}\left(\boldsymbol{e} \cdot \boldsymbol{e}^{\prime}\right)-\left(\boldsymbol{e} \cdot \boldsymbol{w}^{\prime}\right)\left(\boldsymbol{w} \cdot \boldsymbol{e}^{\prime}\right),
\end{aligned}
$$

where $\boldsymbol{w}=\boldsymbol{j} / \sqrt{1-e^{2}}$, and $\boldsymbol{w}^{\prime}=\boldsymbol{j}^{\prime} / \sqrt{1-e^{\prime 2}}$. A quick comparison of (31) and (32) suggests that Milankovitch's formalism is more adapted for this problem. In these variables $\left\langle a^{\prime} / \Delta\right\rangle$, expanded in semimajor ratio, reads

$$
\begin{aligned}
\left\langle\frac{a^{\prime}}{\Delta}\right\rangle_{\lambda, \lambda^{\prime}} & =1+\frac{\alpha^{2}}{8 j^{\prime 5}}\left(3\left(\boldsymbol{j} \cdot \boldsymbol{j}^{\prime}\right)^{2}-\left(1-6 e^{2}\right) j^{2}\right. \\
& \left.-15\left(\boldsymbol{e} \cdot \boldsymbol{j}^{\prime}\right)^{2}\right)+\frac{15 \alpha^{3}}{64 j^{\prime 7}}\left(\left(\boldsymbol{e} \cdot \boldsymbol{e}^{\prime}\right)\right. \\
& \times\left[\left(1-8 e^{2}\right) j^{\prime 2}+35\left(\boldsymbol{e} \cdot \boldsymbol{j}^{\prime}\right)^{2}-5\left(\boldsymbol{j} \cdot \boldsymbol{j}^{\prime}\right)^{2}\right] \\
& \left.-10\left(\boldsymbol{e} \cdot \boldsymbol{j}^{\prime}\right)\left(\boldsymbol{j} \cdot \boldsymbol{e}^{\prime}\right)\left(\boldsymbol{j} \cdot \boldsymbol{j}^{\prime}\right)\right) .
\end{aligned}
$$

One can easily check that $(33)$ is invariant by rotation. The associated equations of motion of the planet and the companion are deduced from (14), 28), and (33) (see Appendix A.2. 
Table 4: Recurrence relations for the computation of $P(k, \ell)$ and $Q(k, \ell)$, Eq. (30).

\begin{tabular}{|c|c|}
\hline$P(k, \ell)=2 P(k-1, \ell-1) P(1,1)-\rho^{4} e^{2} e^{\prime 2} P(k-2, \ell-2)$ & $k \geq 2$ and $\ell \geq 2$ \\
\hline$P(k, 1)=2 P(k-1,0) P(1,1)-\rho^{4} e^{2} Q(k-2,1)$ & $k>2$ \\
\hline$P(1, \ell)=2 P(0, \ell-1) P(1,1)+(-1)^{\ell} \rho^{4} e^{\prime 2} Q(1, \ell-2)$ & $\ell>2$ \\
\hline$P(k, 0)=2(-1)^{k+1} P(k-1,0) P(1,0)+\sigma^{2} \rho^{2} e^{2} P(k-2,0)$ & $k \geq 2$ \\
\hline$P(0, \ell)=2(-1)^{\ell+1} P(0, \ell-1) P(0,1)+\sigma^{2} \rho^{2} e^{\prime 2} P(0, \ell-2)$ & $\ell \geq 2$ \\
\hline \multicolumn{2}{|l|}{$P(2,1)=2 P(1,0) P(1,1)-\rho^{2} e^{2} Q(0,1)$} \\
\hline \multicolumn{2}{|l|}{$P(1,2)=2 P(0,1) P(1,1)+\rho^{2} e^{2} Q(1,0)$} \\
\hline$Q(k, \ell)=2 Q(k-1, \ell-1) Q(1,1)-\sigma^{4} e^{2} e^{\prime 2} Q(k-2, \ell-2)$ & $k>2$ and $\ell \geq 2$ \\
\hline$Q(k, 1)=2 Q(k-1,0) Q(1,1)-\sigma^{4} e^{2} P(k-2,1)$ & $k>2$ \\
\hline$Q(1, \ell)=2 Q(0, \ell-1) Q(1,1)+(-1)^{\ell} \sigma^{4} e^{\prime 2} P(1, \ell-2)$ & $\ell>2$ \\
\hline$Q(k, 0)=P(k, 0), \quad Q(0, \ell)=(-1)^{\ell} P(0, \ell)$ & $\forall k, l$ \\
\hline \multicolumn{2}{|l|}{$Q(2,1)=2 Q(1,0) Q(1,1)-\sigma^{2} e^{2} P(0,1)$} \\
\hline$Q(1,2)=2 Q(0,1) Q(1,1)+\sigma^{2} e^{\prime 2} P(1,0)$ & \\
\hline
\end{tabular}

\subsection{Spin-orbit interaction}

Because of their proper rotations, stars are not spherical and exert a torque on the orbital motion of their planets. Let a system composed of a star $m_{0}$ with one planet $m$. We note $s$ the unit vector along the spin axis of the star, $J_{2}$ its quadrupole gravitational harmonic, and $R_{0}$ its equatorial radius. We make the assumption that $s$ also corresponds to the stellar axis of maximal inertia (gyroscopic approximation). The secular quadrupole potential energy due to the oblateness of the star acting on the planet is (e.g., BL06)

$$
\bar{H}_{\text {spin }}=\frac{\mathcal{G} m_{0} m J_{2} R_{0}^{2}}{4 a^{3}\left(1-e^{2}\right)^{3 / 2}}\left(1-3(\boldsymbol{s} \cdot \boldsymbol{w})^{2}\right) .
$$

This expression is valid as long as the distance of the planet to the star is much larger than the stellar radius $\left(r \gg R_{0}\right)$. Nevertheless, since stellar deformations are usually small, we assume that (34) is valid even for close-in planets with very small semimajor axis. There is no assumption regarding the obliquity of the star relative to the orbital plane of the planet. In a generic problem where $\bar{H}$ represents the Hamiltonian of the system, the equation of motion satisfied by the spin axis is (BL06)

$$
\frac{d s}{d t}=-\frac{1}{L} s \times \nabla_{s} \bar{H},
$$

where $\boldsymbol{L}=C \omega_{0} s$ is the angular momentum of the star, $C$ its moment of inertia along the $s$ axis, and $\omega_{0}$ its rotation rate. The explicit equations of motion of $s, j$ and $\boldsymbol{e}$ are given in Appendix A.3. As shown in BL06, the kinetic energy associated to the rotation of a rigid body around its spin axis, which should be added in the Hamiltonian, does not contribute to the equations of motion once the Hamiltonian is averaged over the proper rotation of the rigid body, or when the body has an axial symmetry. As a consequence, we drop this kinetic energy from our equations. The gravity field coefficient $J_{2}$ is deduced from the rotation speed of the star according to (e.g., Lambeck 1988)

$$
J_{2}=k_{2} \frac{\omega_{0}^{2} R_{0}^{3}}{3 \mathcal{G} m_{0}},
$$

where $k_{2}$ is the second Love number.

\subsection{Relativistic precession}

The last effect taken into account is the secular contribution of general relativity on the precession motion of the planets pericenter. For a planet 
$m$ orbiting a star $m_{0}$, the associated Hamiltonian reads (e.g., Touma et al. 2009)

$$
\bar{H}_{\text {relat }}=-\frac{3 \mu^{2} \beta}{a^{2} c^{2} \sqrt{1-e^{2}}},
$$

where $c$ is the speed of light, $\beta=m_{0} m /\left(m_{0}+m\right)$, and $\mu=\mathcal{G}\left(m_{0}+m\right)$. The associated equations of motion are given in Appendix A.4.

\subsection{Example}

The formalism described above can be used to model many different types of systems. For example, consider a compact planet system perturbed by an outer stellar companion such as the 55 Cancri system (Kaib et al. 2011). If the planet system is similar to those detected by the Kepler spacecraft, it should be dynamically cold with low eccentricities and mutual inclinations. We further assume that the dynamics of the system is not dominated by mean-motion resonances. Conversely, the binary component can be highly eccentric with large inclination with respect to the planet plane. In this case, the Hamiltonian of the system can be approximated by

$$
\begin{aligned}
H_{\text {tot }}= & \sum_{1 \leq j<k \leq p} \bar{H}_{\text {close }}(j, k)+\sum_{j=1}^{p}\left(\bar{H}_{\text {hierar }}(j, p+1)\right. \\
& \left.+\bar{H}_{\text {spin }}(0, j)+\bar{H}_{\text {relat }}(j)\right)
\end{aligned}
$$

where $p$ is the number of planets, and $\bar{H}_{\text {close }}(j, k)$ (26), $\bar{H}_{\text {hierar }}(j, p+1)$ (33), $\bar{H}_{\text {spin }}(0, j)$ (34), and $H_{\text {relat }}(j)$ (37) represent the interactions between planets $j$ and $k$, the interaction between the planet $j$ and the companion, the spin-orbit interaction between the star and the planet $j$, and the relativistic precession of planet $j$, respectively. Naturally, this model is only valid as long as the eccentricity of each planet and the mutual inclination of any pairs of planets remain low. But an increase of any of these two quantities would be the signature of instability and would thus be informative as well.

\section{Numerical tests and applications}

To check our secular models, we performed several tests. First, we considered a system composed of the four inner planets of our solar system. We made this choice because the eccentricities and the mutual inclinations are well known, and relatively low. We used the initial conditions provided in Yoder (1995). This system is used to test the expansions (6) and (26) of the perturbing function in small eccentricity and mutual inclination. In a second step, we chose a hierarchical system undergoing Lidov-Kozai oscillations (Lidov 1962, Kozai 1962 ) to test our expansions in semimajor axis (29) and (33). In both cases, the secular evolutions are compared to numerical simulations done with a full $n$-body symplectic integrator.

\subsection{A compact system}

Figure 2 displays the evolution over one million years of the eccentricities and inclinations of the four planets of our first system with respect to the initial ecliptic plane $\left(I_{\text {Earth }}=0\right.$ at $\left.t=0\right)$ obtained with an $n$-body integrator and by solving the Hamiltonian (26). General relativity is included in both simulations as is the effect of the solar oblateness. The two integrations give very similar results and their distinction is hardly perceptible in most subfigures. It is best seen in the evolution of the eccentricity of Venus and of the Earth, where it consists mostly in a slight shift in the precession frequencies. Thus, the secular formulation preserves the main dynamical features of this system, as already observed by, e.g., Laskar (2008). The integration of the Hamiltonian (6) using Le Verrier's equations of motion is not shown here, because it cannot be distinguished from the solution obtained with the vectorial approach.

In Fig. 3 and 4 , we test the effect of a rotation of the whole system by an angle $\Delta I \in\{0,10,60,90$, $135,180\}$ degrees. More precisely, we apply each of these rotations on the secular integrations only, and we keep the $n$-body integration of the previous figure unchanged. Since the choice of the reference frame is arbitrary, the eccentricities should not be affected by these rotations. Figure 3 shows that this is indeed the case whether the evolution is obtained using Le Verrier's equations 10 or with the vectorial approach (14). On the other hand, Hamiltonians expanded in absolute inclinations are not designed to describe the evolution of such highly inclined systems. It is thus natural to observe discrepancies above $\Delta I=90^{\circ}$ between the secular model taken from, e.g., Laskar \& Robutel (1995) (dotted curve in Fig. 3) and the $n$-body 


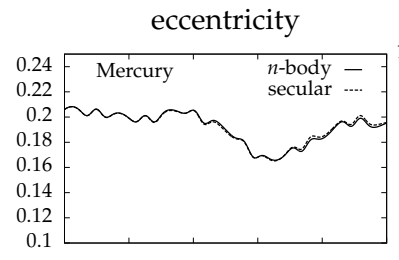

inclination $\left(^{\circ}\right)$
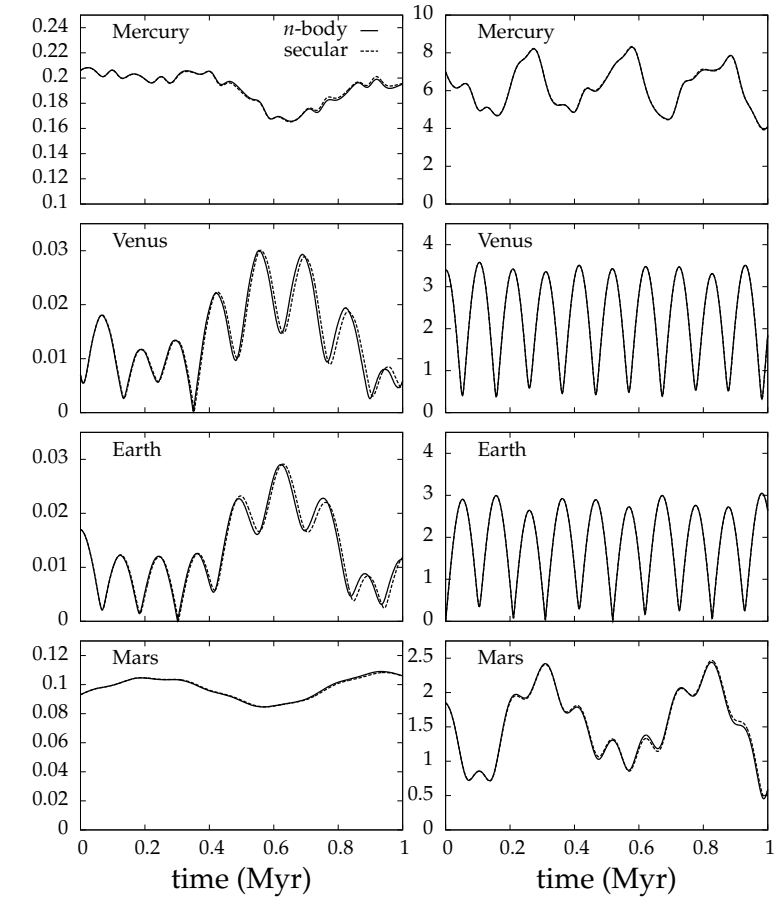

Fig. 2.- Comparison between an $n$-body integration and a secular integration on a system composed of the four inner planets of our solar system followed over one million years.

integration (solid curve). A similar result is observed on Mercury's inclination in Fig. 4. To make this figure, we integrated the system in a tilted frame, and then we applied a rotation of $-\Delta I$ on the output to place the system back in the reference frame of the $n$-body integration. The lack of precision with the expansion in absolute inclination was expected since absolute inclinations are not small as $\Delta I$ increases. However, it is interesting to see that even at $\Delta I=60^{\circ}$, the Hamiltonian expanded in absolute inclination provides reasonable evolutions. This is due to the fact that the system is well described by the Laplace-Lagrange approximation (second order in inclination and eccentricity), and that this approximation is invariant by rotation. Once again, Le Verrier's formalism and the vectorial approach match perfectly, and remain in very good agreement with the full $n$-body integration.

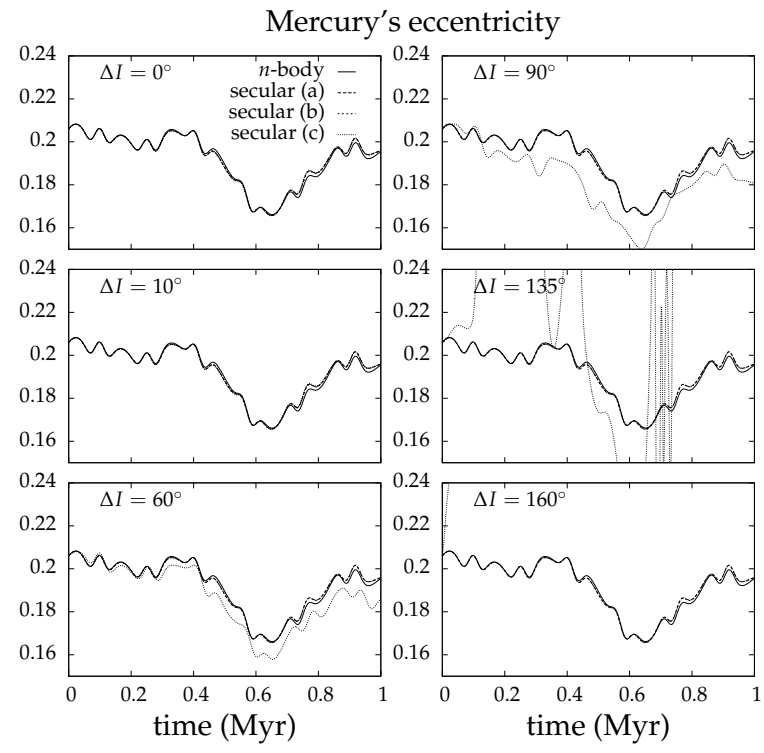

Fig. 3.- Effect of a rotation by $\Delta I$ of the whole system on Mercury's eccentricity simulated with different secular models. The latter are: (a) expansion in eccentricity and mutual inclination in Milankovitch's variables, (b) expansion in eccentricity and mutual inclination using Le Verrier's equations, and (c) expansion in eccentricity and absolute inclination (Laskar \& Robutel 1995). Models (a) and (b) overlap and cannot be distinguished from one another.

\subsection{Lidov-Kozai oscillations}

To test our expansions in semimajor axis 29 and (33), we integrate a system composed of a planet with mass $m_{1}=1 M_{J}$, semimajor axis $a_{1}=6 \mathrm{au}$, and initial eccentricity $e_{1}=0.001$ and a brown dwarf with mass $m_{2}=40 M_{J}$, semimajor axis $a_{2}=100 \mathrm{au}$, and initial eccentricity $e_{2}=0.6$. The mass of the central star is $m_{0}=1 M_{\odot}$, and the mutual inclination is initially set to $J=65^{\circ}$. With these values, the system undergoes Lidov-Kozai oscillations whose modeling requires the octupole order (Naoz et al. 2011). The integrations are performed with an $n$-body code, and with the secular approximations 29. and (33). In all simulations general relavity (37) is included. The results are displayed Fig. 5 . The two secular models are strictly equivalent, the solutions are thus indistinguishable. This was not the case for the Hamiltonian expanded in mutual 

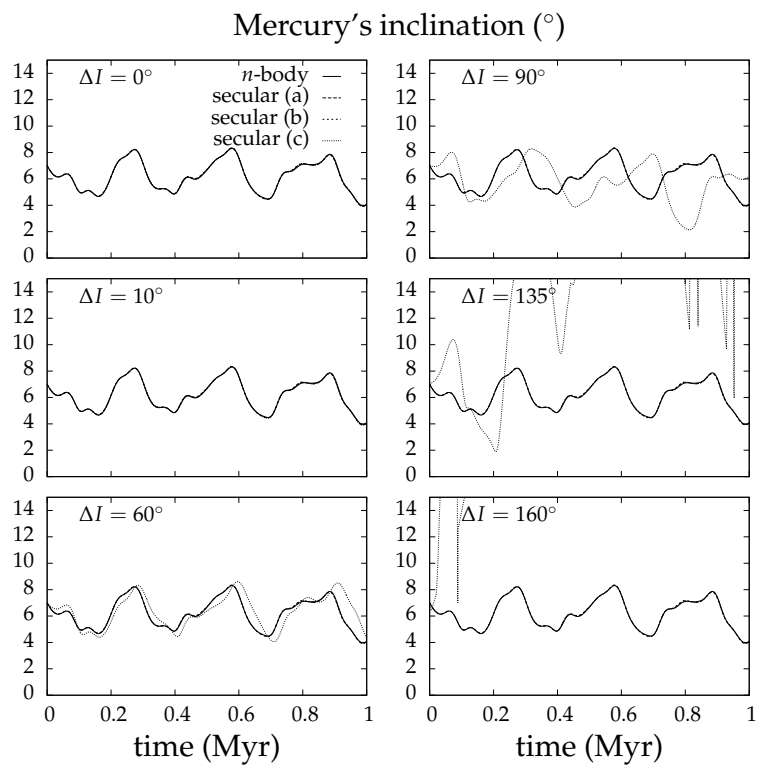

Fig. 4.- Same as Fig. 3 but with Mercury's inclination. In all panels, inclinations are computed with respect to the same reference plane after performing a rotation of $-\Delta I$ on the output of each simulation. Models (a) and (b) are overlapping and cannot be distinguished from one another.

inclination and eccentricities since the quantities $p(k, \ell)$ and $q(k, \ell)$ Eq. 22, present in the Hamiltonian (6), were themselves expanded in $T_{1}, T_{2}$, $V_{1}, V_{2}, W_{1}$, and $W_{2}$ to get the expression (26). The comparison with the $n$-body integration is also very good. The integrations differ slightly once the planet flips into a retrograde orbit. This transition does not occur at the exact same time in the $n$-body and the secular simulations, as a result, the direction of the ninth kick in the evolution of $I_{1}$ is not the same in the two integrations. Nevertheless, the transition is preceded by a passage through an extreme and unrealistic eccentricity where the periastron distance, $0.06 R_{\odot}$, is much shorter than the size of the central star.

\section{Conclusion}

We have provided a vectorial formalism for studying the secular motion of non-resonant conservative gravitational systems with concentric orbits such as planetary systems. All expressions are analytical and expressed in terms of the vectors $\boldsymbol{e}$ and $\boldsymbol{j}$, namely the Laplace-Runge-Lenz vec-
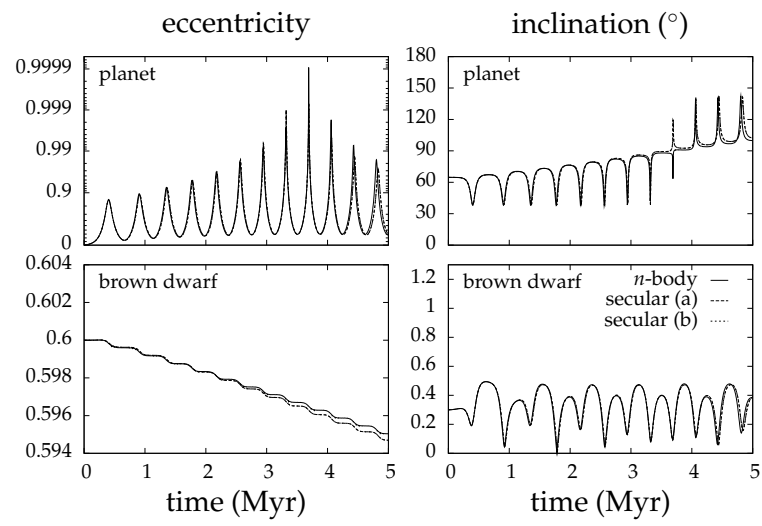

Fig. 5.- Comparison between an $n$-body simulation and secular integrations of a hierarchical three-body system. The parameters are taken from (Naoz et al. 2011, fig. 1). The system is composed of a planet with mass $m_{1}=1 M_{J}$, at $a_{1}=6$ au with an initial eccentricity of $e_{1}=0.001$, and a brown dwarf with mass $m_{2}=40 M_{J}$, at $a_{2}=100$ au and an initial eccentricity $e_{2}=0.6$. The initial mutual inclination is $J=65^{\circ}$. The secular models are: (a) expansion in eccentricity and mutual inclination in Milankovitch's variables and (b) expansion in eccentricity and mutual inclination using Le Verrier's equations. Note that the eccentricity of the planet (upper left panel) is plotted in a $\tanh ^{-1}$ scale.

tor and the dimensionless orbital angular momentum. Planet-planet interactions have been developed either in eccentricity and mutual inclination or in semimajor axis ratio using an algorithm adapted from Abdullah (2001). For completeness, the vectorial expression of the spin-orbit interaction and general relativity have also been recalled. With numerical tests, we have shown that the integrations of the vectorial equations are in perfect agreement with the more standard approach relying on classical elliptic elements $(a, e, I, \lambda, \varpi, \Omega)$. The Kepler spacecraft has revealed a population of low-inclination, low-mass, and low-period planets which are not readily studied with N-body techniques. The present formalism can be used to study their dynamics, including the onset of secular chaos. Moreover, the vectorial approach naturally is expanded to include a companion on a hierarchical orbit. That companion could be a binary star companion, or a giant planet at a con- 
siderable distance. As long as the inner planet system remains with coplanar orbits and low eccentricities, our formalism remains valid. It can, in fact, be used to see whether the system will remain stable, or whether secular chaos will lead to orbit crossings, which then must be followed with an N-body approach. Inclusion of the precession dynamics of central star also allows us to address the problem of spin-orbit alignment in multiplanet systems, which is newly observationally relevant, and which we will pursue in a follow-up paper (Boué and Fabrycky, 2014).

GB thanks Philippe Robutel, Jacques Laskar and Alain Albouy for the many discussions which have been helpful for this study, and in particular those about Khaled Adbulah's PhD thesis. 


\section{A. Explicit vectorial equations of motion}

In this appendix, we provide the explicit expressions of the secular equations of motion written in a vectorial form. Notations are the same as in Section 2.1.2. We consider both compact and hierarchical systems. In each case, the parameters of the outer body are noted with a prime, while those of the inner body are unprimed.

\section{A.1. Compact system}

The Hamiltonian (26) describing the secular evolution of a compact two planet system reads

$$
\begin{aligned}
H_{1}= & -\frac{\mathcal{G} m m^{\prime}}{a^{\prime}}\left[c_{1}+c_{2}\left(e^{2}+e^{\prime 2}+\boldsymbol{j} \cdot \boldsymbol{j}^{\prime}-1\right)+c_{3}\left(\boldsymbol{e} \cdot \boldsymbol{e}^{\prime}\right)+c_{4} e^{2} e^{2}+c_{5}\left(\alpha^{2} e^{4}+e^{\prime 4}\right)+c_{6}\left(\boldsymbol{e} \cdot \boldsymbol{e}^{\prime}\right)^{2}\right. \\
& +c_{7}\left(1-\boldsymbol{j} \cdot \boldsymbol{j}^{\prime}\right)^{2}+c_{8}\left(\boldsymbol{e} \cdot \boldsymbol{j}^{\prime}\right)^{2}+c_{9}\left(\boldsymbol{j} \cdot \boldsymbol{e}^{\prime}\right)^{2}+c_{10}\left(\alpha\left(1-\boldsymbol{j} \cdot \boldsymbol{j}^{\prime}\right) e^{2}+\left(\boldsymbol{e} \cdot \boldsymbol{e}^{\prime}\right) e^{\prime 2}\right) \\
& \left.+c_{11}\left(\left(1-\boldsymbol{j} \cdot \boldsymbol{j}^{\prime}\right) e^{\prime 2}+\alpha\left(\boldsymbol{e} \cdot \boldsymbol{e}^{\prime}\right) e^{2}\right)+c_{12}\left(\left(1-\boldsymbol{j} \cdot \boldsymbol{j}^{\prime}\right)\left(\boldsymbol{e} \cdot \boldsymbol{e}^{\prime}\right)-\left(\boldsymbol{e} \cdot \boldsymbol{j}^{\prime}\right)\left(\boldsymbol{j} \cdot \boldsymbol{e}^{\prime}\right)\right)\right]
\end{aligned}
$$

where the $c_{i}$ 's are parameters depending on Laplace coefficients (see Tab. 3). The conservation of the orbital angular momentum implies that

$$
\Lambda \frac{d \boldsymbol{j}}{d t}=-\Lambda^{\prime} \frac{d \boldsymbol{j}^{\prime}}{d t^{\prime}}=\boldsymbol{T}_{1}
$$

where $\boldsymbol{T}_{1}=-\boldsymbol{j} \times \nabla_{\boldsymbol{j}} H_{1}-\boldsymbol{e} \times \boldsymbol{\nabla}_{\boldsymbol{e}} H_{1}$ is a torque whose expression is

$$
\boldsymbol{T}_{1}=\frac{\mathcal{G} m m^{\prime}}{a^{\prime}}\left(A_{1} \boldsymbol{j} \times \boldsymbol{j}^{\prime}+B_{1} \boldsymbol{e} \times \boldsymbol{e}^{\prime}+C_{1} \boldsymbol{e} \times \boldsymbol{j}^{\prime}+D_{1} \boldsymbol{j} \times \boldsymbol{e}^{\prime}\right) .
$$

The other equations of motion, deduced from (14), are

$$
\frac{d \boldsymbol{e}}{d t}=\frac{\mathcal{G} m m^{\prime}}{a^{\prime} \Lambda}\left(A_{1} \boldsymbol{e} \times \boldsymbol{j}^{\prime}+B_{1} \boldsymbol{j} \times \boldsymbol{e}^{\prime}+C_{1} \boldsymbol{j} \times \boldsymbol{j}^{\prime}+D_{1} \boldsymbol{e} \times \boldsymbol{e}^{\prime}+E_{1} \boldsymbol{j} \times \boldsymbol{e}\right),
$$

and

$$
\frac{d \boldsymbol{e}^{\prime}}{d t}=\frac{\mathcal{G} m m^{\prime}}{a^{\prime} \Lambda^{\prime}}\left(A_{1} \boldsymbol{e}^{\prime} \times \boldsymbol{j}+B_{1} \boldsymbol{j}^{\prime} \times \boldsymbol{e}+C_{1} \boldsymbol{e}^{\prime} \times \boldsymbol{e}+D_{1} \boldsymbol{j}^{\prime} \times \boldsymbol{j}+F_{1} \boldsymbol{j}^{\prime} \times \boldsymbol{e}^{\prime}\right)
$$

with

$$
\begin{aligned}
& A_{1}=c_{2}-2 c_{7}\left(1-\boldsymbol{j} \cdot \boldsymbol{j}^{\prime}\right)-\alpha c_{10} e^{2}-c_{11} e^{2}-c_{12}\left(\boldsymbol{e} \cdot \boldsymbol{e}^{\prime}\right), \\
& B_{1}=c_{3}+2 c_{6}\left(\boldsymbol{e} \cdot \boldsymbol{e}^{\prime}\right)+c_{10} e^{2}+\alpha c_{11} e^{2}+c_{12}\left(1-\boldsymbol{j} \cdot \boldsymbol{j}^{\prime}\right), \\
& C_{1}=2 c_{8}\left(\boldsymbol{e} \cdot \boldsymbol{j}^{\prime}\right)-c_{12}\left(\boldsymbol{j} \cdot \boldsymbol{e}^{\prime}\right), \\
& D_{1}=2 c_{9}\left(\boldsymbol{j} \cdot \boldsymbol{e}^{\prime}\right)-c_{12}\left(\boldsymbol{e} \cdot \boldsymbol{j}^{\prime}\right), \\
& E_{1}=2 c_{2}+2 c_{4} e^{\prime 2}+4 \alpha^{2} c_{5} e^{2}+2 \alpha c_{10}\left(1-\boldsymbol{j} \cdot \boldsymbol{j}^{\prime}\right)+2 \alpha c_{11}\left(\boldsymbol{e} \cdot \boldsymbol{e}^{\prime}\right), \\
& F_{1}=2 c_{2}+2 c_{4} e^{2}+4 c_{5} e^{\prime 2}+2 c_{10}\left(\boldsymbol{e} \cdot \boldsymbol{e}^{\prime}\right)+2 c_{11}\left(1-\boldsymbol{j} \cdot \boldsymbol{j}^{\prime}\right) .
\end{aligned}
$$

\section{A.2. Hierarchical system}

The Hamiltonian (33) describing the secular evolution of a hierarchical two planet system reads

$$
\begin{aligned}
H_{2}= & -\frac{\mathcal{G} m m^{\prime}}{a^{\prime}}\left[1+\frac{\alpha^{2}}{8 j^{\prime 5}}\left(3\left(\boldsymbol{j} \cdot \boldsymbol{j}^{\prime}\right)^{2}-\left(1-6 e^{2}\right) j^{\prime 2}-15\left(\boldsymbol{e} \cdot \boldsymbol{j}^{\prime}\right)^{2}\right)\right. \\
& \left.+\frac{15 \alpha^{3}}{64 j^{\prime 7}}\left(\left[\left(1-8 e^{2}\right) j^{\prime 2}+35\left(\boldsymbol{e} \cdot \boldsymbol{j}^{\prime}\right)^{2}-5\left(\boldsymbol{j} \cdot \boldsymbol{j}^{\prime}\right)^{2}\right]\left(\boldsymbol{e} \cdot \boldsymbol{e}^{\prime}\right)-10\left(\boldsymbol{e} \cdot \boldsymbol{j}^{\prime}\right)\left(\boldsymbol{j} \cdot \boldsymbol{e}^{\prime}\right)\left(\boldsymbol{j} \cdot \boldsymbol{j}^{\prime}\right)\right)\right],
\end{aligned}
$$


where $j=\|\boldsymbol{j}\|=\sqrt{1-e^{2}}$. The conservation of the orbital angular momentum implies that

$$
\Lambda \frac{d \boldsymbol{j}}{d t}=-\Lambda^{\prime} \frac{d \boldsymbol{j}^{\prime}}{d t}=\boldsymbol{T}_{2}
$$

where $\boldsymbol{T}_{2}=-\boldsymbol{j} \times \boldsymbol{\nabla}_{\boldsymbol{j}} H_{2}-\boldsymbol{e} \times \boldsymbol{\nabla}_{\boldsymbol{e}} H_{2}$ is a torque whose expression is

$$
\boldsymbol{T}_{2}=\frac{\mathcal{G} m m^{\prime}}{a^{\prime}}\left(A_{2} \boldsymbol{j} \times \boldsymbol{j}^{\prime}+B_{2} \boldsymbol{e} \times \boldsymbol{e}^{\prime}+C_{2} \boldsymbol{e} \times \boldsymbol{j}^{\prime}+D_{2} \boldsymbol{j} \times \boldsymbol{e}^{\prime}\right) .
$$

The other equations of motion, deduced from (14), are

$$
\frac{d \boldsymbol{e}}{d t}=\frac{\mathcal{G} m m^{\prime}}{a^{\prime} \Lambda}\left(A_{2} \boldsymbol{e} \times \boldsymbol{j}^{\prime}+B_{2} \boldsymbol{j} \times \boldsymbol{e}^{\prime}+C_{2} \boldsymbol{j} \times \boldsymbol{j}^{\prime}+D_{2} \boldsymbol{e} \times \boldsymbol{e}^{\prime}+E_{2} \boldsymbol{j} \times \boldsymbol{e}\right),
$$

and

$$
\frac{d \boldsymbol{e}^{\prime}}{d t}=\frac{\mathcal{G} m m^{\prime}}{a^{\prime} \Lambda^{\prime}}\left(A_{2} \boldsymbol{e}^{\prime} \times \boldsymbol{j}+B_{2} \boldsymbol{j}^{\prime} \times \boldsymbol{e}+C_{2} \boldsymbol{e}^{\prime} \times \boldsymbol{e}+D_{2} \boldsymbol{j}^{\prime} \times \boldsymbol{j}+F_{2} \boldsymbol{j}^{\prime} \times \boldsymbol{e}^{\prime}\right),
$$

with

$$
\begin{aligned}
A_{2}= & \frac{3 \alpha^{2}}{4 j^{\prime 5}}\left(\boldsymbol{j} \cdot \boldsymbol{j}^{\prime}\right)-\frac{75 \alpha^{3}}{32 j^{\prime 7}}\left[\left(\boldsymbol{e} \cdot \boldsymbol{j}^{\prime}\right)\left(\boldsymbol{j} \cdot \boldsymbol{e}^{\prime}\right)+\left(\boldsymbol{e} \cdot \boldsymbol{e}^{\prime}\right)\left(\boldsymbol{j} \cdot \boldsymbol{j}^{\prime}\right)\right] \\
B_{2}= & \frac{15 \alpha^{3}}{64 j^{\prime 7}}\left[\left(1-8 e^{2}\right) j^{\prime 2}+35\left(\boldsymbol{e} \cdot \boldsymbol{j}^{\prime}\right)^{2}-5\left(\boldsymbol{j} \cdot \boldsymbol{j}^{\prime}\right)^{2}\right], \\
C_{2}= & -\frac{15 \alpha^{2}}{4 j^{\prime 5}}\left(\boldsymbol{e} \cdot \boldsymbol{j}^{\prime}\right)+\frac{75 \alpha^{3}}{32 j^{\prime 7}}\left[7\left(\boldsymbol{e} \cdot \boldsymbol{j}^{\prime}\right)\left(\boldsymbol{e} \cdot \boldsymbol{e}^{\prime}\right)-\left(\boldsymbol{j} \cdot \boldsymbol{e}^{\prime}\right)\left(\boldsymbol{j} \cdot \boldsymbol{j}^{\prime}\right)\right], \\
D_{2}= & -\frac{75 \alpha^{3}}{32 j^{\prime 7}}\left(\boldsymbol{e} \cdot \boldsymbol{j}^{\prime}\right)\left(\boldsymbol{j} \cdot \boldsymbol{j}^{\prime}\right), \\
E_{2}= & \frac{3 \alpha^{2}}{2 j^{\prime 3}}-\frac{15 \alpha^{3}}{4 j^{\prime 5}}\left(\boldsymbol{e} \cdot \boldsymbol{e}^{\prime}\right), \\
F_{2}= & \frac{3 \alpha^{2}}{8 j^{\prime 7}}\left[5\left(\boldsymbol{j} \cdot \boldsymbol{j}^{\prime}\right)^{2}-\left(1-6 e^{2}\right) j^{\prime 2}-25\left(\boldsymbol{e} \cdot \boldsymbol{j}^{\prime}\right)^{2}\right] \\
& +\frac{75 \alpha^{3}}{64 j^{\prime 9}}\left(\left[\left(1-8 e^{2}\right) j^{\prime 2}+49\left(\boldsymbol{e} \cdot \boldsymbol{j}^{\prime}\right)^{2}-7\left(\boldsymbol{j} \cdot \boldsymbol{j}^{\prime}\right)^{2}\right]\left(\boldsymbol{e} \cdot \boldsymbol{e}^{\prime}\right)-14\left(\boldsymbol{e} \cdot \boldsymbol{j}^{\prime}\right)\left(\boldsymbol{j} \cdot \boldsymbol{e}^{\prime}\right)\left(\boldsymbol{j} \cdot \boldsymbol{j}^{\prime}\right)\right) .
\end{aligned}
$$

\section{A.3. Spin-orbit interaction}

The Hamiltonian (34) governing the spin-orbit evolution of a planet orbiting an oblate star is

$$
H_{3}=\frac{G m_{0} m J_{2} R_{0}^{2}}{4 a^{3}\left(1-e^{2}\right)^{3 / 2}}\left(1-3(\boldsymbol{s} \cdot \boldsymbol{w})^{2}\right) .
$$

Once again, the conservation of the angular momentum implies that

$$
L \frac{d s}{d t}=-\Lambda \frac{d \boldsymbol{j}}{d t}=\boldsymbol{T}_{3},
$$

with $\boldsymbol{T}_{3}=-\boldsymbol{s} \times \boldsymbol{\nabla}_{\boldsymbol{s}} H_{3}$ is the torque acting on the stellar rotation. Its expression is

$$
\boldsymbol{T}_{3}=\frac{3}{2} \frac{G m_{0} m J_{2} R_{0}^{2}}{a^{3}\left(1-e^{2}\right)^{3 / 2}}(\boldsymbol{s} \cdot \boldsymbol{w}) \boldsymbol{s} \times \boldsymbol{w} .
$$

The evolution of the eccentricity vector $e$, deduced from (14), is given by

$$
\Lambda \sqrt{1-e^{2}} \frac{d \boldsymbol{e}}{d t}=-\frac{3}{2} \frac{G m_{0} m J_{2} R_{0}^{2}}{a^{3}\left(1-e^{2}\right)^{3 / 2}}\left[(\boldsymbol{s} \cdot \boldsymbol{w}) \boldsymbol{s} \times \boldsymbol{e}+\frac{1}{2}\left(1-5(\boldsymbol{s} \cdot \boldsymbol{w})^{2}\right) \boldsymbol{w} \times \boldsymbol{e}\right] .
$$




\section{A.4. General relativity}

The effect of general relativity induced by the massive central star is modeled by the Hamiltonian (37)

$$
H_{4}=-\frac{3 \mu^{2} \beta}{a^{2} c^{2} \sqrt{1-e^{2}}} .
$$

The orbital angular momentum of the planet is conserved, and its eccentricity vector evolves according to

$$
\frac{d \boldsymbol{e}}{d t}=\frac{3 \mu^{2} \beta}{a^{2} c^{2} \Lambda\left(1-e^{2}\right)} \boldsymbol{w} \times \boldsymbol{e} .
$$




\section{REFERENCES}

Abdullah, K. 2001, PhD thesis, Observatoire de Paris,

www.imcce.fr/public/theses/abdullah_these.ps

Albrecht, S., Winn, J. N., Johnson, J. A., et al. 2012, ApJ, 757, 18

Batygin, K. 2012, Nature, 491, 418

Batygin, K., Morbidelli, A., \& Tsiganis, K. 2011, A\&A, 533, A7

Beaugé, C., \& Nesvorný, D. 2012, ApJ, 751, 119

Boué, G., \& Laskar, J. 2006, Icarus, 185, 312

—. 2009, Icarus, 201, 750

Breiter, S., \& Ratajczak, R. 2005, MNRAS, 364, 1222

Correia, A. C. M., Laskar, J., Farago, F., \& Boué, G. 2011, Celestial Mechanics and Dynamical Astronomy, 111, 105

Duquennoy, A., \& Mayor, M. 1991, A\&A, 248, 485

Ellis, K. M., \& Murray, C. D. 2000, Icarus, 147, 129

Fabrycky, D., \& Tremaine, S. 2007, ApJ, 669, 1298

Fabrycky, D. C., Lissauer, J. J., Ragozzine, D., et al. 2012, ArXiv e-prints, arXiv:1202.6328

Farago, F., \& Laskar, J. 2010, MNRAS, 401, 1189

Figueira, P., Marmier, M., Boué, G., et al. 2012, A\&A, 541, A139

Fischer, D. A., Marcy, G. W., Butler, R. P., et al. 2008, ApJ, 675, 790

Gastineau, M., \& Laskar, J. 2012, TRIP 1.2.26, TRIP Reference manual, IMCCE, Paris Observatory, http://www.imcce.fr/trip/

Hansen, P. A. 1853, Abhandl. d. K. S. Ges. d. Wissensch., IV, 182

Hill, G. W. 1875, The Analyst, 2, 161

Holman, M., Touma, J., \& Tremaine, S. 1997, Nature, 386,254
Innanen, K. A., Zheng, J. Q., Mikkola, S., \& Valtonen, M. J. 1997, AJ, 113, 1915

Kaib, N. A., Raymond, S. N., \& Duncan, M. J. 2011, ApJ, 742, L24

Katz, B., Dong, S., \& Malhotra, R. 2011, Physical Review Letters, 107, 181101

Kozai, Y. 1962, AJ, 67, 591

Kratter, K. M., \& Perets, H. B. 2012, ApJ, 753, 91

Lai, D. 2014, MNRAS, 440, 3532

Lambeck, K. 1988, Geophysical geodesy : the slow deformations of the earth (Oxford [England] : Clarendon Press ; New York : Oxford University Press, 1988.)

Laskar, J. 1984, Thèse, Observatoire de Paris http://tel.archives-ouvertes.fr/ tel-00702723

—. 1989, Nature, 338, 237

—. 1990, Icarus, 88, 266

—. 2008, Icarus, 196, 1

Laskar, J., \& Boué, G. 2010, A\&A, 522, A60

Laskar, J., \& Robutel, P. 1995, Celestial Mechanics and Dynamical Astronomy, 62, 193

Le Verrier, U. J. J. 1855, Annales de l'Observatoire impérial de Paris: mémoires (Impr. GauthierVillars)

Li, G., Naoz, S., Kocsis, B., \& Loeb, A. 2014, ApJ, 785,116

Libert, A.-S., \& Delsate, N. 2012, MNRAS, 422, 2725

Libert, A.-S., \& Henrard, J. 2007, Icarus, 191, 469

-. 2008, Celestial Mechanics and Dynamical Astronomy, 100, 209

Libert, A.-S., \& Tsiganis, K. 2009, MNRAS, 400, 1373

—. 2011, MNRAS, 412, 2353

Lidov, M. L. 1962, Planet. Space Sci., 9, 719 
Lithwick, Y., \& Naoz, S. 2011, ApJ, 742, 94

Lithwick, Y., \& Wu, Y. 2011, ApJ, 739, 31

Mardling, R. A. 2010, MNRAS, 407, 1048

Michtchenko, T. A., Ferraz-Mello, S., \& Beaugé, C. 2006, Icarus, 181,555

Migaszewski, C., \& Goździewski, K. 2009, MNRAS, 395, 1777

Milankovitch, M. 1939, Bull. Serb. Acad. Math. Nat. A, 6, 1

Morbidelli, A., Tsiganis, K., Batygin, K., Crida, A., \& Gomes, R. 2012, Icarus, 219, 737

Mugrauer, M., Neuhäuser, R., Mazeh, T., et al. 2006, Astronomische Nachrichten, 327, 321

Nagasawa, M., \& Ida, S. 2011, ApJ, 742, 72

Naoz, S., Farr, W. M., Lithwick, Y., Rasio, F. A., \& Teyssandier, J. 2011, Nature, 473, 187

-. 2013a, MNRAS, 431, 2155

Naoz, S., Farr, W. M., \& Rasio, F. A. 2012, ApJ, 754, L36

Naoz, S., Kocsis, B., Loeb, A., \& Yunes, N. 2013b, ApJ, 773, 187

Petrovich, C. 2014, ArXiv e-prints, arXiv: 1405.0280

Raghavan, D., McAlister, H. A., Henry, T. J., et al. 2010, ApJS, 190, 1

Rosengren, A. J., \& Scheeres, D. J. 2014, Celestial Mechanics and Dynamical Astronomy, 118, 197

Saleh, L. A., \& Rasio, F. A. 2009, ApJ, 694, 1566

Souriau, J. M. 1969, Structure des systèmes dynamiques (Dunod, Paris)

Takeda, G., Kita, R., \& Rasio, F. A. 2008, ApJ, 683, 1063

Terquem, C., \& Papaloizou, J. C. B. 2002, MNRAS, 332, L39

Tisserand, F. 1889, Traité de Mécanique Céleste (Paris: Gauthier-Villars)
Touma, J. R., Tremaine, S., \& Kazandjian, M. V. 2009, MNRAS, 394, 1085

Tremaine, S., \& Dong, S. 2012, AJ, 143, 94

Tremaine, S., Touma, J., \& Namouni, F. 2009, AJ, 137, 3706

Tremaine, S., \& Yavetz, T. 2013, ArXiv e-prints, arXiv:1309.5244

Triaud, A. H. M. J. 2011, A\&A, 534, L6

Triaud, A. H. M. J., Collier Cameron, A., Queloz, D., et al. 2010, A\&A, 524, A25

Udry, S., \& Santos, N. C. 2007, ARA\&A, 45, 397

Veras, D., \& Tout, C. A. 2012, MNRAS, 422, 1648

Winn, J. N., Fabrycky, D., Albrecht, S., \& Johnson, J. A. 2010, ApJ, 718, L145

Wu, Y., \& Lithwick, Y. 2011, ApJ, 735, 109

—. 2013, ApJ, 772, 74

Wu, Y., \& Murray, N. 2003, ApJ, 589, 605

Wu, Y., Murray, N. W., \& Ramsahai, J. M. 2007, ApJ, 670, 820

Yoder, C. F. 1995, in Global Earth Physics: A Handbook of Physical Constants, ed. T. J. Ahrens, 1

Zucker, S., \& Mazeh, T. 2002, ApJ, 568, L113

This 2-column preprint was prepared with the AAS LATEX macros v5.2. 